\title{
LA CONSECUCIÓN DE LA IGUALDAD DE MUJERES Y HOMBRES EN EL ESPACIO EUROPEO DE INVESTIGACIÓN: DEL INFORME ETAN AL PROGRAMA MARCO DE INVESTIGACIÓN E INNOVACIÓN HORIZONTE EUROPA
}

The achievement of equality of women and men in the European Research Area: From the ETAN Report to the Framework Programme for Research and Innovation Horizon Europe

\author{
MARÍA NIEVES SALDAÑA DÍAZ \\ Universidad de Huelva \\ manieves@uhu.es
}

Cómo citar/Citation Saldaña Díaz, M. N. (2021). La consecución de la igualdad de mujeres y hombres en el Espacio Europeo de Investigación: del Informe Etan al Programa Marco de Investigación e Innovación Horizonte Europa. lgualdadES, 4, 43-85 doi: https://doi.org/10.18042/cepc/lgdES.4.02

(Recepción: 30/06/2020; aceptación tras revisión: 19/04/2021; publicación: 30/06/2021)

\section{Resumen}

Desde la publicación del conocido Informe ETAN en 2000, la política de la Unión Europea en el ámbito de la investigación y la innovación, articulada fundamentalmente a través del Espacio Europeo de Investigación, incluye entre sus prioridades la igualdad de mujeres y hombres y la incorporación de la perspectiva de género en la investigación, definiéndose acciones para abordar los desequilibrios de género en las instituciones de investigación e integrar la dimensión de género y el análisis de sexo/género en el contenido de la investigación, como refleja el Programa Marco de Investigación e Innovación Horizonte 2020, así como el Programa Marco 
Horizonte Europa (2021-2027). No obstante, para asegurar su plena eficacia será indispensable que la Unión Europea adopte una directiva específica que obligue a todos los Estados miembros a adoptar disposiciones legislativas de carácter vinculante en sus estrategias nacionales de investigación e innovación, incluyéndose sistemas eficaces de seguimiento y evaluación.

\title{
Palabras clave
}

Unión Europea; Espacio Europeo de Investigación; igualdad de mujeres y hombres; perspectiva de género.

\begin{abstract}
Since the publication of the well-known ETAN Report in 2000, the policy of the European Union in the area of research and innovation, mainly articulated through the European Research Area, includes among its prioirities the equality between women and men and the incorporation of the gender perspective in research, defining actions to address gender imbalances in research institutions and integrating the gender dimension and the analysis of sex/gender in the content of research, as reflected in the Framework Program for Research and Innovation Horizon 2020, as well as the Framework Program Horizon Europe (2021-2027). However, in order to ensure its full effectiveness, it will be essential for the European Union itself to adopt a specific Directive that obliges all the States to adopt binding legislative provisions in their national research and innovation strategies, including effective systems of monitoring and evaluation.
\end{abstract}

\section{Keywords}

European Union; European Research Area; equality of women and men; gender perspective. 


\section{SUMARIO}

I. INTRODUCCIÓN. II. LA IGUALDAD DE MUJERES Y HOMBRES EN EL ESPACIO EUROPEO DE INVESTIGACIÓN (ERA): DEL INFORME ETAN A LA INCORPORACIÓN DE LA PERSPECTIVA DE GÉNERO CON CARÁCTER TRANSVERSAL (1999-2014). III. IMPLEMENTACIÓN DE LA DIMENSIÓN DE GÉNERO EN LA INVESTIGACIÓN EN EL PROGRAMA MARCO HORIZONTE 2020 (2014-2020). IV. EVALUACIÓN DE LA INCORPORACIÓN DE LA PERSPECTIVA DE GÉNERO EN HORIZONTE 2020. V. HACIA LA CONSECUCIÓN DE LA IGUALDAD DE MUJERES Y HOMBRES Y LA INTEGRACIÓN DE LA DIMENSIÓN DE GÉNERO EN LA INVESTIGACIÓN EN EL PROGRAMA MARCO HORIZONTE EUROPA (2021-2027). VI. CONCLUSIONES: LA NECESARIA ADOPCIÓN DE UNA DIRECTIVA RELATIVA A IGUALDAD DE MUJERES Y HOMBRES E INTEGRACIÓN DE LA PERSPECTIVA Y DIMENSIÓN DE GÉNERO EN EL ERA. BIBLIOGRAFía.

\section{INTRODUCCIÓN}

La Unión Europea (UE) ha venido desarrollando a lo largo de más de sesenta años un marco regulatorio específico para la consecución de la igualdad de mujeres y hombres ${ }^{1}$. Ya el Tratado constitutivo de las Comunidades Europeas (TCCE) de 1957 instauró una prohibición de discriminación por razón de sexo en materia salarial, estableciéndose un principio de igualdad de retribuciones entre trabajadores masculinos y femeninos para un mismo trabajo (art. 119 TCCE) ${ }^{2}$. Con el Tratado de Ámsterdam (TUE) de 1997 se establece como objetivo eliminar las desigualdades entre el hombre y la mujer, adoptándose un enfoque dual, consistente, por una parte, en la integración del principio de igualdad de género en todas las políticas comunitarias, la llamada transversalidad de género (gender mainstreaming), y por otra, en la aplicación de medidas específicas (acción positiva) a favor de las mujeres (arts. 2, 3, 13, 137.1, 141.1 TUE) 3 . Asimismo, la Carta de Derechos Fundamentales de la

\footnotetext{
Parlamento Europeo (2020).

2 Tratado de Roma. Tratado constitutivo de la Comunidad Económica Europea, de 25 de marzo de 1957 (no publicado en el Diario Oficial).

3 DO C 340 de 10-11-1997. Disponible en: https://cutt.ly/obUwFfx.
} 
Unión Europea (CDFUE), adoptada en Niza el 7 de diciembre de 2000, que ha adquirido carácter jurídicamente vinculante tras la entrada en vigor del Tratado de Lisboa (art. 6.1 TUE), prohíbe toda discriminación por razón de sexo y afirma que la igualdad de mujeres y hombres debe garantizarse en todas las áreas, inclusive en materia de empleo, trabajo y remuneración (arts. 21 y 23 CDFUE) ${ }^{4}$. Y tras la adopción del vigente Tratado de Lisboa en 2007, la Unión Europea combate la discriminación y fomenta la igualdad entre mujeres y hombres (art. 3.3 TUE); fija como objetivo eliminar las desigualdades entre el hombre y la mujer y promover su igualdad (art. 8 TFUE); lucha contra toda discriminación por razón de sexo en la definición y ejecución de sus políticas y acciones (arts. 10 y 19 TFUE); apoya y completa la acción de los Estados miembros en el ámbito de la igualdad entre mujeres y hombres por lo que respecta a las oportunidades en el mercado laboral y al trato en el trabajo (art. 153.1.i); establece el principio de igualdad de retribución sin discriminación por razón de sexo por un mismo trabajo o por un trabajo de igual valor, debiendo ser garantizado por cada Estado miembro (art. 157.1 TFUE); dispone el principio de igualdad de oportunidades e igualdad de trato para hombres y mujeres en asuntos de empleo y ocupación, que debe ser garantizado por el Parlamento Europeo y el Consejo (art. 157.3 TFUE), y reconoce que los Estados puedan adoptar medidas de acción positiva destinadas a facilitar al sexo menos representado el ejercicio de actividades profesionales o a evitar o compensar desventajas en sus carreras profesionales (art. 157.4 TFUE) $)^{5}$.

Sobre la base de este marco normativo, y especialmente a partir de la década de 1970, la Unión Europea ha adoptado más de una decena de directivas, que son vinculantes para los Estados miembros y deben ser objeto de transposición a la legislación nacional, para garantizar la igualdad de trato y oportunidades entre mujeres y hombres y la prohibición de discriminación por razón de sexo, especialmente en materia de igualdad de retribuciones, igualdad de trato en lo que se refiere al acceso al empleo, a la formación y a la promoción profesionales, condiciones de trabajo, seguridad social, trabajadores autónomos, protección de la maternidad, acceso a bienes y servicios y su suministro y la conciliación de la vida familiar y la vida profesional de los progenitores y los cuidadores ${ }^{6}$. Asimismo,

DO C 364 de 18-12-2000. Disponible en: https://bit.ly/2R6iva6.

DO C 202 de 7-6-2016. Disponible en: https://cutt.ly/8bUwMmO.

6 Véase Directiva 92/85/CEE del Consejo, de 19 de octubre de 1992, relativa a la aplicación de medidas para promover la mejora de la seguridad y de la salud en el trabajo de la trabajadora embarazada, que haya dado a luz o esté en período de lactancia, DO L 348 de 28-11-1992; Directiva 97/81/CE del Consejo, de 15 de diciembre de 1997, 
la Comisión Europea ha impulsado la adopción de programas plurianuales y distintas iniciativas para promover la igualdad de mujeres y hombres. Así, desde 1982, en que se aprobó por la Comisión el I Programa de Acción Comunitario para la Promoción de la Igualdad de Oportunidades para Mujeres y Hombres (1982-1985), se han adoptado sucesivos programas comunitarios (1986-1990, 1991-1995, 1996-2000, 2001-2005, 2006-2010, 2011-2015 у 2016-2019) que configuran un marco de referencia en relación con diversas áreas prioritarias consideradas estratégicas para avanzar en la igualdad entre mujeres y hombres tanto a nivel europeo como estatal ${ }^{7}$. Actualmente, la vigente Estrategia para la Igualdad de Género 2020-2025, adoptada por la Comisión el 5 de marzo de 2020, se centra en seis objetivos estratégicos: poner fin a la violencia de género y combatir los estereotipos de género; prosperar en una economía con igualdad de género colmando las brechas de género en el mercado de trabajo, logrando la participación en pie de igualdad en los distintos sectores de la economía, abordando la brecha salarial y de pensiones entre hombres y mujeres y colmando la brecha de género en las responsabilidades asistenciales; alcanzar la igualdad en

relativa al Acuerdo marco sobre el trabajo a tiempo parcial concluido por la UNICE, el CEEP y la CES, DO L 14 de 20-1-1998; Directiva del Consejo 2004/113/CE, de 13 de diciembre de 2004, por la que se aplica el principio de igualdad de trato entre hombres y mujeres al acceso a bienes y servicios y su suministro, DO L 373 de 21-122004; Directiva 2006/54/CE del Parlamento Europeo y del Consejo, de 5 de julio de 2006, relativa a la aplicación del principio de igualdad de oportunidades e igualdad de trato entre hombres y mujeres en asuntos de empleo y ocupación, DO L 204 de 26-7-2006; Directiva 2010/41/UE del Parlamento Europeo y del Consejo, de 7 de julio de 2010, sobre la aplicación del principio de igualdad de trato entre hombres y mujeres que ejercen una actividad autónoma, y por la que se deroga la Directiva 86/613/CEE del Consejo, DO L 180 de 15-7-2010; y Directiva 2019/1158/UE del Parlamento Europeo y del Consejo, de 20 de junio de 2019, relativa a la conciliación de la vida familiar y la vida profesional de los progenitores y los cuidadores, y por la que se deroga la Directiva 2010/18/UE del Consejo, DO L 188 de 12-7-2019.

7 Para coadyuvar a la consecución de los objetivos y acciones previstas en los programas, estrategias y planes de acción, en 2006 se creó el Instituto Europeo de Igualdad de Género (European Institute For Gender Equality, EIGE por sus siglas en inglés), que presta asistencia técnica a las instituciones de la Unión y a los Estados miembros, llevando a cabo diversas funciones de recopilación, análisis y difusión de datos e implementación de herramientas metodológicas en materia de igualdad entre mujeres y hombres. Todos los estudios, publicaciones e instrumentos pueden consultarse en su web institucional, disponible en: https://eige.europa.eu/. Entre las diversas áreas sobre las que el Instituto Europeo de Igualdad de Género centra sus actuaciones se incluye la relativa a investigación, cuya información está disponible en: https:/eige.europa. eu/topics/research. 
los puestos de mando en todos los ámbitos de la sociedad, logrando el equilibrio de género en la toma de decisiones y la política; integrar la perspectiva de género e interseccional en las políticas de la UE; adoptar acciones de financiación para avanzar en la igualdad de género en el UE, y abordar la igualdad de género y el empoderamiento de las mujeres en todo el mundo.

Conforme a tal marco de referencia y especialmente a partir del Informe $E T A N^{9}$, la política comunitaria en el ámbito de la investigación y la innovación $(\mathrm{I}+\mathrm{i})^{10}$ (arts. 179-190 TFUE) $)^{11}$, articulada fundamentalmente

8 Comunicación de la Comisión al Parlamento Europeo, al Consejo, al Comité Económico y Social Europeo y al Comité de las Regiones, de 5 de marzo de 2020, relativa a una «Unión de la Igualdad: Estrategia para la Igualdad de Género 2020-2025», COM (2020) 152 final. Disponible en: https://cutt.ly/nbUej0d.

9 Comisión Europea (2000).

10 Aunque en 1957 el Tratado Constitutivo de la Comunidad Económica y Europea dio lugar a una serie de programas de investigación en los temas prioritarios de aquel momento, como la energía, la biotecnología o el medio ambiente, no sería hasta 1983 cuando se establece el Primer Programa Marco de Investigación, centrado en materias relacionadas con las telecomunicaciones, la biotecnología y la tecnología industrial (1983-1987). Tres años más tarde, el 17 de febrero de 1986, la investigación se conforma como política comunitaria al incluirse un título VI específico relativo a la investigación y el desarrollo tecnológico en el Acta Única Europea (AUE), con el objetivo de fortalecer las bases científicas y tecnológicas de la industria europea y favorecer el desarrollo de su competitividad internacional, estimulándose a las universidades, centros de investigación y empresas en sus esfuerzos de investigación y desarrollo tecnológico y previéndose la aplicación de programas marcos plurianuales adoptados por el Consejo por unanimidad, DO Suplemento 2/86. Disponible en: https://bit.ly/3xvTW7q.

11 El título XIX del vigente Tratado de Funcionamiento de la Unión Europea (TFUE) define la política comunitaria en el ámbito de la investigación y el desarrollo tecnológico (arts. 179-190). Los objetivos generales son fortalecer las bases científicas y tecnológicas del tejido investigador y empresarial europeo y favorecer el desarrollo de su competitividad internacional, así como fomentar todas las acciones de investigación que se realicen en virtud de los Tratados (art. 179 TFUE). Para la consecución de tales objetivos, las acciones de la Unión se centran fundamentalmente en la ejecución de programas de investigación, desarrollo tecnológico y demostración, promoviéndose la cooperación con las empresas, los centros de investigación y las universidades, y el estímulo a la formación y movilidad del personal investigador de la Unión (art. 180 TFUE). Para su ejecución se contempla la aprobación por el Parlamento y el Consejo, con arreglo al procedimiento legislativo ordinario y previa consulta al Comité Económico y Social, de un programa marco plurianual que fija los objetivos científicos y tecnológicos que deben alcanzarse mediante las acciones contempladas en 
a través del Espacio Europeo de Investigación (European Research Area, ERA por sus siglas en inglés $)^{12}$, incluye entre sus prioridades la igualdad de mujeres y hombres y la incorporación de la perspectiva de género en la investigación (Biglia y Vergés-Bosch, 2016), definiéndose acciones para abordar los desequilibrios de género en las instituciones de investigación y órganos de toma de decisiones e integrar la perspectiva de género, la dimensión de género y el análisis de sexo/género en el contenido de la investigación, implementadas especialmente en los programas marcos plurianuales de investigación e innovación ${ }^{13}$.

Como ha analizado la Comisión Europea, la participación de las mujeres en la ciencia y la tecnología contribuye a aumentar la calidad, la relevancia social y la competitividad de la investigación y la innovación y, específicamente, el equilibrio de género en la toma de decisiones contribuye a avanzar en la consecución de la igualdad de oportunidades entre mujeres y hombres

el art. 180 TFUE y las prioridades correspondientes, fija el importe global máximo y la participación financiera de la Unión en el Programa Marco, ejecutándose mediante programas específicos desarrollados dentro de cada una de las acciones (art. 182 TFUE), DO C de 26-10-2012. Disponible en: https://cutt.ly/JbUeQYX.

12 En la Comunicación de la Comisión al Consejo, al Parlamento Europeo, al Comité Económico y Social y al Comité de las Regiones, de 18 de enero de 2000, "Hacia un Espacio Europeo de Investigación", se inicia el proceso de configuración del ERA, articulándose en torno a un triple objetivo: la creación de un mercado interior de investigación, dirigido a reforzar la cooperación, estimular la competencia y sacar el máximo partido a la asignación de los recursos; una reestructuración del tejido europeo de investigación, consistente fundamentalmente en una mejor coordinación de las actividades y políticas de investigación nacionales, ya que estas representan la mayor parte de la investigación realizada y financiada en Europa; y el fomento de una política europea de investigación que vaya más allá de la mera financiación de las actividades de investigación y que incluya todos los aspectos de las demás políticas nacionales y europeas relacionadas con la misma. Para la consecución de tales objetivos, el ERA incluye, entre otros elementos: establecimiento de una red de los centros de excelencia científica existente en Europa y creación de centros virtuales utilizando las nuevas herramientas de comunicación interactivas; enfoque común de las necesidades y medios de financiación de las grandes infraestructuras de investigación; establecimiento de un sistema común de referencia científico y técnico para la aplicación de las políticas; incremento de la movilidad del personal investigador e introducción de una dimensión europea en las carreras científicas; refuerzo del estatuto y el papel de las mujeres en la investigación; y promoción de valores sociales y éticos comunes en materia científica y tecnológica, $\mathrm{COM}(2000) 6$ final, 7-8. Disponible en: https://cutt.ly/JbUeT6g.

13 Consejo de la Unión Europea (2015a). 
y a ampliar el alcance de las políticas de investigación e innovación y, por lo tanto, a atender mejor las necesidades de todos los sectores de la sociedad ${ }^{14}$. Asimismo, la calidad de la investigación se ve afectada cuando la dimensión de género no se tiene en cuenta, pues el género es una variable analítica y explicativa clave en la investigación, al ser resultado de un proceso sociocultural que se articula en torno a los valores culturales y las actitudes sociales que, en conjunto, dan forma y sancionan los comportamientos de mujeres y hombres, afectando a los productos, las tecnologías, los entornos y el conocimiento. De ahí que los estereotipos de género a menudo no se cuestionen, influyendo inconscientemente en las prioridades científicas, los métodos y resultados de investigación. En consecuencia, es necesario integrar la dimensión de género en la investigación, teniéndose en cuenta en todo el proceso de investigación, al desarrollar conceptos y teorías, formular preguntas de investigación, recopilar y analizar datos así como al utilizar las herramientas analíticas apropiadas para cada campo científico, coadyuvándose así a repensar los estándares y los modelos científicos de referencia que discriminan a las mujeres. Por tanto, la integración de la dimensión de género contribuye a la excelencia investigadora, incrementando la relevancia social del conocimiento y de las tecnologías e innovaciones, a cuya consecución se dirige el ERA ${ }^{15}$.

Sin embargo, como se constata en este estudio, a pesar de haber transcurrido ya más de veinte años desde la publicación el Informe ETAN, los progresos han sido lentos e insuficientes, estando todavía afectada la investigación por un conjunto de desigualdades de género persistentes, especialmente la segregación de género en investigación y ciencia, los retos en la carrera profesional relacionados con el género, el desequilibrio de género en cargos superiores en la academia, los sesgos de género en el acceso a la financiación de la investigación, la investigación ciega al género y sesgada en el género y la cultura organizativa y el proceso institucional ciego al género y con sesgo de género ${ }^{16}$, como muestran los datos publicados en She Figures $2018^{17}$, así como las resistencias constantes a la implementación efectiva de transversalización de género (Vida, 2020; Mergaert y Lombardo, 2014), como reflejan los procesos de evaluación del Sexto Programa Marco (2002-2006), el Séptimo Programa Marco (2007-2013) y Horizonte 2020 (2014-2020), de ahí que haya llegado el momento de que la UE adopte disposiciones legislativas vinculantes que garanticen avanzar de forma más efectiva durante la vigencia del Programa

\footnotetext{
14 Comisión Europea (2018).

15 Comisión Europea (2020: 8); Advisory Group for Gender (2016: 2, 2015: 2).

16 European Institute for Gender Equality (2016a).

17 Comisión Europea (2019c).
} 
Marco Horizonte Europa (2021-2027) en la consecución de la igualdad de mujeres y hombres, así como en la transversalización de la perspectiva de género y la integración de la dimensión de género en el ERA.

\section{LA IGUALDAD DE MUJERES Y HOMBRES EN EL ESPACIO EUROPEO DE INVESTIGACIÓN: DEL INFORME ETAN A LA INCORPORACIÓN DE LA PERSPECTIVA DE GÉNERO CON CARÁCTER TRANSVERSAL (1999-2014)}

La promoción de la igualdad de mujeres y hombres ha formado parte del enfoque estratégico de la Comisión Europea en el marco del ERA, especialmente a partir de 1999. En efecto, ya la Comunicación de la Comisión al Consejo, al Parlamento Europeo, al Comité Económico y Social y al Comité de las Regiones, de 18 de enero de 2000, «Hacia un Espacio Europeo de Investigación", por la que se inicia el proceso de configuración del ERA, destaca como medida de acción específica el refuerzo del estatuto y papel de las mujeres en la investigación ${ }^{18}$. Realmente, un año antes la Comisión Europea formaliza su compromiso de promover la igualdad de género en la investigación en la Comunicación de la Comisión, de 17 de febrero de 1999, relativa a «Mujeres y ciencia: movilizar a las mujeres en beneficio de la investigación europea», en la que se propone una estrategia coherente dentro del Quinto Programa Marco de Investigación, Desarrollo Tecnológico y Demostración (1998-2002) para promover la investigación por, para y sobre las mujeres (Dewandre, 2002), estableciéndose dos objetivos específicos: estimular el debate y el intercambio de experiencias en este campo entre los Estados miembros para que se puedan tomar medidas de la manera más eficaz posible y desarrollar un enfoque coherente con el objetivo de aumentar significativamente el número de mujeres que participan en la investigación, señalándose como objetivo lograr al menos un $40 \%$ de participación de mujeres en todos los niveles de aplicación y gestión de los programas de investigación, especialmente en las becas de investigación Marie Curie, en los grupos de asesoramiento y en los paneles de evaluación del Quinto Programa Marco de Investigación ${ }^{19}$.

Unos meses más tarde, se adopta la Resolución del Consejo, de 20 de mayo de 1999, sobre "La mujer y la ciencia», en la que se reconoce la infrarrepresentación de las mujeres en el ámbito de la investigación científica y técnica, instándose a los Estados a facilitar la información existente sobre el

18 COM (2000) 6 final, 8. Disponible en: https://cutt.ly/dbO4zja.

19 COM (1999) 76 final. Disponible en: https://bit.ly/3nAYTr6. 
equilibrio de género en el personal dedicado a investigación y a la propia Comisión a presentar datos comparables e indicadores europeos que sirvan de base para evaluar a nivel comunitario la situación de la mujer en el ámbito de la investigación y proseguir los esfuerzos para alentar una mayor participación de la mujer en todo el Quinto Programa $\mathrm{Marco}^{20}$. Y, posteriormente, se adopta la Resolución del Parlamento Europeo, de 3 de febrero de 2000, sobre la Comunicación de la Comisión Mujeres y Ciencia: «Movilizar a las mujeres en beneficio de la investigación europea», en la que se insta tanto a la Comisión como a los Estados miembros a adoptar diversas medidas que coadyuven a alcanzar los objetivos marcados en la Comunicación de la Comisión ${ }^{21}$.

Desde entonces, la promoción de la igualdad de mujeres y hombres ha sido parte del enfoque estratégico de la Comisión Europea en el marco del ERA. Así, el Grupo de Trabajo sobre las Mujeres y la Ciencia de la Red Europea de Evaluación de Tecnología (European Technology Assessment Network, ETAN), publica en el año 2000 el pionero Informe sobre politica cientifica de la Unión Europea. Promover la excelencia mediante la integración de la igualdad entre géneros (conocido como Informe ETAN), que presenta un estudio estadístico sobre la situación de las mujeres en la educación superior, los institutos de investigación así como en los comités científicos, tanto a nivel europeo como de los distintos Estados miembros, recomendándose, entre otras medidas: la mejora y armonización de la dimensión de género en las bases de datos de la Unión Europea; una participación mínima de cada género en los comités científicos del $30 \%$ en 2002 y del $40 \%$ en 2005; integrar la igualdad de género en el Sexto Programa Marco y en los programas de financiación de la ciencia y la tecnología de los Estados miembros; la supervisión, el seguimiento y la utilización de incentivos económicos para asegurar los avances en igualdad de género, y la participación activa de la Comisión Europea, el Parlamento Europeo, el Consejo y las instituciones de los Estados miembros que financian y gestionan la ciencia (Rees, 2001)22.

Por tanto, el ERA debe contribuir a alcanzar tales objetivos reforzando la presencia de las mujeres en la investigación mediante la acción conjunta de los Estados y de la propia Unión Europea. Como señala la Comunicación de la Comisión al Consejo, al Parlamento Europeo, al Comité Económico y Social y al Comité de las Regiones, de 18 de enero de 2000, «Hacia un Espacio Europeo de Investigación», en el año 2000 las mujeres no están suficientemente

20 DO C 201 de 16-07-1999. Disponible en: https://cutt.ly/ZbO4BvS.

21 COM (1999) 76-C5-0103/1999-1999/2106 (COS). Disponible en: https://cutt.ly/ QbO40kE.

22 Comisión Europea (2000). 
presentes en la investigación en Europa, pues aunque representan un 50\% del total de los licenciados universitarios e incluso sobrepasan en número a los hombres en algunas disciplinas (las ciencias y tecnologías de la vida, por ejemplo), sin embargo, no se encuentran en la misma proporción en los laboratorios y servicios de investigación de las universidades, su progresión en la carrera científica es más lenta que la de los hombres y su número decrece a medida que se sube en la escala de responsabilidades, habiendo en la cúspide de la jerarquía académica de los Estados de la Unión, por término medio, menos de un $10 \%$ de mujeres. En el origen de esta situación se encuentran varios mecanismos de discriminación, así como la escasa consideración de las dificultades particulares a las que deben enfrentarse para desarrollar sus actividades profesionales, constituyendo una pérdida para las propias mujeres, para la investigación y para la sociedad ${ }^{23}$.

Tres años más tarde, en la Comunicación de la Comisión al Consejo y al Parlamento Europeo, de 18 de julio de 2003, relativa a «Los investigadores en el Espacio Europeo de Investigación: una profesión con múltiples carreras», se aborda de manera específica las desigualdades entre hombres y mujeres en las actividades de $\mathrm{I}+\mathrm{D}$, debiendo interpretarse los reducidos porcentajes de participación global de las mujeres conforme al conocido fenómeno de leaky pipeline (cañería que gotea), según el cual el número de mujeres tiende a disminuir de manera desproporcionada a medida que se avanza hacia los puestos más altos del escalafón, representando menos del $10 \%$ de los titulares de cátedras de los Estados de la UE, proponiéndose para su superación que las carreras de $\mathrm{I}+\mathrm{D}$ dejen de considerarse un obstáculo para las obligaciones familiares, que sigue afectando de manera casi exclusiva a las mujeres, y que se reconozcan los logros que alcanzan las mujeres, reconociéndose y teniéndose en cuenta los distintos impactos que las carreras de $\mathrm{I}+\mathrm{D}$ tienen sobre las mujeres y los hombres ${ }^{24}$.

Posteriormente, en la Comunicación de la Comisión al Parlamento Europeo, al Consejo, al Comité Económico y Social Europeo y al Comité de las Regiones, de 17 de julio de 2012, relativa a «Una asociación del Espacio Europeo de Investigación reforzada en pos de la excelencia y el crecimiento», se pretende incrementar la eficacia, eficiencia y excelencia de la investigación europea, definiéndose entre las prioridades del ERA la igualdad de mujeres y hombres y la integración de la perspectiva de género en la investigación, pues, a a pesar de las estrategias nacionales, a nivel europeo la investigación europea sigue adoleciendo de una pérdida considerable y un uso ineficiente

23 COM (2000) 6 final, 17. Disponible en: https://cutt.ly/jbO7ytd.

${ }^{24} \mathrm{COM}$ (2003) 436 final. Disponible en: https://cutt.ly/CbO7uHk. 
de las mujeres altamente cualificadas, siendo demasiado pocas las que ocupan posiciones de liderazgo o participan en la toma de decisiones. Así, aunque en 2005 el Consejo fijó el objetivo de que las mujeres ocupasen el $25 \%$ de los principales puestos de investigación del sector público, sin embargo, en 2009 tan solo el $13 \%$ de las personas responsables de instituciones de enseñanza superior eran mujeres. Asimismo, la integración de la dimensión del género en el diseño, evaluación y ejecución de la investigación sigue siendo bastante limitada. Por tanto, es necesario mejorar en todos estos puntos para incrementar la calidad y la pertinencia de la investigación, sin olvidar que la Comisión ya se había comprometido a garantizar un $40 \%$ de personas del sexo menos representado en todos sus grupos de personal experto, paneles y comités, teniendo previsto aplicar este compromiso especialmente en el marco de Horizonte $2020^{25}$.

Como se señala en el documento que evalúa el impacto del ERA y que acompaña a la Comunicación de la Comisión, no todos los Estados miembros tienen políticas nacionales para alentar el uso del talento potencial de las mujeres científicas y la integración de una dimensión de género en el contenido de la investigación. Esto implica que el potencial cualificado de las científicas sea infrautilizado y que las mujeres estén infrarrepresentadas, faltando el equilibrio de género en la toma de decisiones en investigación e innovación, y que la falta de una dimensión de género en el contenido de la investigación socave la calidad y la relevancia de las actividades de investigación. Y sin mejorar la participación de las mujeres y la integración de la dimensión de género en el contenido de la investigación, los objetivos del ERA de ofrecer altos niveles de empleo, productividad y cohesión social no serán totalmente alcanzables, pues una mayor participación de las mujeres en la investigación contribuirá al crecimiento socioeconómico europeo, así como también al impacto en el rendimiento de la investigación y la excelencia en la investigación, que son objetivos fundamentales del ERA ${ }^{26}$.

Por todo ello, en la Comunicación de la Comisión, de 17 de julio de 2012, relativa a «Una asociación del Espacio Europeo de Investigación reforzada en pos de la excelencia y el crecimiento", se insta a los Estados miembros a la implementación de diversas medidas, especialmente: ofrecer incentivos para eliminar los obstáculos jurídicos y de otro tipo que dificultan la contratación, retención y promoción profesional de las investigadoras; combatir los desequilibrios de género en los procesos de toma de decisiones; reforzar la perspectiva de género en los programas de investigación, y velar por que al menos un

25 COM (2012) 392 final. Disponible en: https://bit.ly/32SCXy9.

26 Comisión Europea (2012a: 13). 
$40 \%$ de personas del sexo menos representado participen en los comités que intervienen en la contratación/promoción en la carrera del personal y en el establecimiento y evaluación de los programas de investigación. Asimismo, se insta a las universidades e instituciones de investigación a aplicar modificaciones institucionales a través de planes de igualdad de mujeres y hombres que tengan por finalidad llevar a cabo evaluaciones de impacto o auditorías de los procedimientos y prácticas con el fin de detectar sesgos por motivos de género, aplicar estrategias para corregir cualquier sesgo y fijar objetivos y efectuar un seguimiento de los avances por medio de indicadores. Finalmente, la propia Comisión Europea se compromete a fomentar la igualdad de mujeres y hombres y la integración de la perspectiva de género en los proyectos y programas de Horizonte 2020 desde su inicio hasta su evaluación ${ }^{27}$.

Tresaños mástarde, en las «Conclusionessobreel avancedela igualdad de género en el Espacio Europeo de Investigación", adoptadas el 1 de diciembre de 2015, el Consejo insta a los Estados miembros y las organizaciones de investigación a adoptar medidas para garantizar que la asignación de financiación para investigación no se vea afectada por ningún sesgo de género, a promover condiciones de trabajo flexibles que posibiliten la conciliación profesional y familiar, a revisar el desempeño del personal investigador para eliminar posibles sesgos de género, y a promover objetivos cuantitativos para lograr un equilibrio de género equitativo en los niveles académicos superiores del profesorado universitario, en los órganos de adopción de decisiones, incluidos los principales comités científicos y administrativos, los comités de contratación y promoción, así como en los paneles de evaluación, alentando alcanzar tales objetivos en $2020^{28}$.

Ese mismo año, a instancias del Consejo de la Unión Europea ${ }^{29}$, se aprueba la Hoja de ruta del ERA 2015-2020, identificándose un número concreto de prioridades de implementación clave que contribuyan a alcanzar el mayor impacto posible en los sistemas europeos de ciencia, investigación e innovación, instando el Consejo a los Estados miembros y a la Comisión Europea a aplicar las prioridades de acción identificadas en la Hoja de ruta, especialmente, por lo que aquí interesa, la prioridad 4, centrada en la igualdad de género e incorporación de la dimensión de género en la investigación, y que se dirige expresamente a la legislación nacional para que se adopten medidas efectivas que aborden los desequilibrios de género en las instituciones de investigación y los órganos de toma de decisiones e integren

27 COM (2012) 392 final, 15. Disponible en: https://bit.ly/2R7aWQM.

28 Consejo de la Unión Europea (2015a).

29 Consejo de la Unión Europea (2014). 
mejor la dimensión de género en las políticas, programas y proyectos de investigación ${ }^{30}$. Habiéndose evaluado específicamente la implementación de la igualdad de mujeres y hombres, así como la integración de la perspectiva de género en las políticas, programas y proyectos de $\mathrm{I}+\mathrm{i}$ en los informes de progreso del ERA publicados a partir de $2012^{31}$.

\section{IMPLEMENTACIÓN DE LA DIMENSIÓN DE GÉNERO EN LA INVESTIGACIÓN EN EL PROGRAMA MARCO HORIZONTE 2020 (2014-2020)}

Para coadyuvar a la ejecución de las acciones previstas en el ERA, en 2014 se pone en marcha el Programa Marco de Investigación e Innovación Horizonte 2020, que constituye el mayor programa marco de este ámbito implementado por la Unión Europea hasta la fecha, con un período de vigencia previsto de siete años (2014-2020) y un presupuesto de casi 80000 millones de euros. El nuevo programa, que se articula en torno a tres pilares fundamentales, «ciencia excelente», «liderazgo industrial» y «retos de la sociedad», reúne por primera vez todos los fondos europeos destinados a la investigación e innovación con reglas comunes para todos los participantes, proporcionando financiación en todas las etapas del proceso de innovación e investigación, contribuyendo así a la implementación del ERA en el marco de un mercado único del conocimiento, la investigación y la innovación ${ }^{32}$.

Como se viene analizando, fomentar la igualdad de género en la investigación y la innovación es un compromiso del ERA, de ahí que Horizonte 2020 aborde el género como una cuestión transversal para corregir las disparidades entre mujeres y hombres e integrar la perspectiva de género en el contenido y la programación de la investigación y la innovación ${ }^{33}$ (Díaz y

30 Consejo de la Unión Europea (2015b).

31 Comisión Europea (2013a; 2014; 2017a; 2019b).

32 Véase Decisión del Consejo, de 3 de diciembre de 2013, por la que se establece el programa específico por el que se ejecuta «Horizonte 2020: Programa Marco de Investigación e Innovación (2014-2020)» y se derogan las Decisiones 2006/971/ CE, 2006/972/CE, 2006/973/CE, 2006/974/CE y 2006/975/CE (2013/743/UE), DO L 347/965 de 20-12-2013, párr. 976. Disponible en: https://cutt.ly/lbO5aay; Declaración de la Comisión (Programa Marco) (2013/C 373/02), DO L 373/12 de 20-12-2013. Disponible en: https://bit.ly/3vriLzv.

33 Decisión del Consejo, de 3 de diciembre de 2013, por la que se establece el programa específico por el que se ejecuta «Horizonte 2020: Programa Marco de Investigación e Innovación (2014-2020)» y se derogan las Decisiones 2006/971/CE, 2006/972/CE, 
Samper, 2014). Así, el art. 16 del Reglamento Marco establece que Horizonte 2020 garantizará la promoción eficaz de la igualdad entre hombres y mujeres y de la dimensión de género en el contenido de la investigación y la innovación, prestándose especial atención a garantizar el equilibrio de género en los equipos de investigación, grupos de evaluación y grupos consultivos y grupos de personal experto, integrándose la dimensión de género adecuadamente en el contenido de investigación e innovación de las estrategias, programas y proyectos, y haciendo un seguimiento de la misma en todas las etapas del ciclo de investigación. Y el art. 14 sobre cuestiones transversales añade que se establecerán vínculos e interfaces entre las prioridades de Horizonte 2020, prestándose especial atención, entre ellas, a las cuestiones de género ${ }^{34}$.

Por tanto, la perspectiva de género está incluida en los documentos centrales que configuran Horizonte 2020 atendiendo a tres objetivos principales: el equilibrio de género en los equipos de investigación y en la toma de decisiones y la integración del análisis de sexo/género en el contenido de la investigación e innovación. Por lo que se refiere al equilibrio de género en los equipos de investigación, se pretende alcanzar el objetivo establecido por la Comisión del $40 \%$ del sexo menos representado en cada grupo (personal experto, etc.) y paneles (evaluación, etc.), elevándose para los grupos asesores al $50 \%$, dada la alta tasa de respuesta de las mujeres a la convocatoria de expresiones de interés de la Comisión lanzada en febrero de 2013, debiendo cada grupo incluir al menos una persona experta con experiencia en género. En cuanto al equilibrio de género en la toma de decisiones, se fomenta una participación equilibrada de mujeres y hombres en las actividades de investigación de las diferentes etapas del ciclo, debiendo incluirse el equilibrio de género en el equipo de investigación entre los factores de clasificación para priorizar las propuestas con las mismas puntuaciones. Y en lo referente a la integración del análisis de sexo/género en el contenido de la investigación e innovación, la dimensión de género está explícitamente integrada en varios temas en todas las secciones del programa de trabajo de Horizonte 2020, debiendo exponer cada solicitante cómo se tiene en cuenta el análisis de género en el contenido del proyecto cuando el tema y/o sus conclusiones puedan afectar a las personas

2006/973/CE, 2006/974/CE y 2006/975/CE (2013/743/UE), DO L $347 / 965$ de 20-12-2013, párr. 976. Disponible en: https://cutt.ly/lbO5aay.

34 Reglamento (UE) n. ${ }^{\circ}$ 1291/2013 del Parlamento Europeo y del Consejo, de 11 de diciembre de 2013 por el que se establece «Horizonte 2020: Programa Marco de Investigación e Innovación (2014-2020)», y por el que se deroga la Decisión n. ${ }^{\circ}$ 1982/2006/CE, DO L 347/104 de 20-12-2013. Disponible en: https://bit.ly/2R8rSpL. 
por razón de su sexo, pues el reconocimiento de las diferencias de género puede tener implicaciones importantes para el conocimiento científico. La integración de la dimensión de género en la investigación e innovación ha sido objeto de análisis en distintos proyectos financiados en el marco del Séptimo Programa Marco y en Horizonte 2020. Así, el Grupo de Expertos en Innovaciones de Género de la Comisión Europea ha desarrollado métodos prácticos de análisis de sexo/género especialmente en los campos de ciencias básicas, ingeniería y desarrollo tecnológico, medio ambiente, alimentación y nutrición, salud y medicina, transporte y ciencias de la comunicación ${ }^{35}$. Asimismo, la herramienta en línea GEAR (Gender Equality in Academia and Research), desarrollada por el Instituto Europeo de Igualdad de Género, analiza con detenimiento cómo la integración de la dimensión de género en el contenido de la investigación contribuye a la excelencia, incluyéndose casos concretos extraídos de nueve campos de investigación específicos ${ }^{36}$. Igualmente, GENDER-NET (2013-2016) ha desarrollado un manual relativo a la integración del análisis de sexo/género en el contenido de la investigación (GENDER-NET, 2016). Actualmente, el proyecto GENDER-NET PLUS: Promoting Gender Equality in H2020 and the ERA (2017-2022) está dirigido a fortalecer a las entidades gestoras de programas de investigación y agencias de financiación para fomentar la integración del análisis de sexo/género en la investigación y en los programas de investigación ${ }^{37}$. Y el Proyecto SUPERA (Supporting the Promotion of Equality in Research and Academia) (2018-2022) tiene como principal objetivo el desarrollo de planes de igualdad en cuatro universidades europeas y dos agencias de financiación que coadyuven a articular políticas de igualdad efectivas, así como la inclusión de la perspectiva de género en la investigación ${ }^{38}$.

Estos tres objetivos se ajustan a los establecidos en la Comunicación de la Comisión de julio de 2012 sobre la realización del ERA, integrándose en cada etapa del ciclo de investigación e innovación. Además, el área transversal relativa a "Ciencia con y para la sociedad» de Horizonte 2020 incluye como actividad específica promover la «igualdad de género en investigación e innovación» (GERI, por sus siglas en inglés), especialmente apoyando cambios estructurales de la organización de las instituciones de investigación y en el contenido y diseño de las actividades investigadoras con el objetivo de llegar a una masa crítica de universidades e instituciones de investigación europeas

\footnotetext{
35 Comisión Europea (2013b).

36 European Institute for Gender Equality (2016a).

37 Disponible en: http://gender-net-plus.eu/.

38 Disponible en: https://www.superaproject.eu/.
} 
que implementen cambios institucionales a través de planes de igualdad de género $^{39}$. Asimismo, una novedad de Horizonte 2020 es la inclusión de la capacitación en cuestiones de género entre los costes elegibles de una acción, con el objetivo de ayudar al personal investigador a desarrollar y compartir experiencias de género en relación con el proyecto financiado ${ }^{40}$.

Los objetivos establecidos en materia de igualdad de género en Horizonte 2020 son necesarios, como muestra la evaluación ex-post del Séptimo Programa Marco (FP7 por sus siglas en inglés) (2007-2013), evidenciándose que la igualdad de género en investigación e innovación avanza muy lentamente. Así, durante el FP7 el porcentaje de mujeres coordinadoras de proyectos aumentó ligeramente del $16-17 \%$ en 2006 al 19,2\% en 2012, representando las mujeres el $38 \%$ de la fuerza de trabajo total informada de los proyectos. Sin embargo, solo el $29 \%$ de los puestos de liderazgo y el $34 \%$ del personal investigador con experiencia eran mujeres, aunque entre el personal doctor involucrado en el FP7 las mujeres representaban el 45\%. Esta segregación vertical también se combina con una horizontal, con una proporción de mujeres que depende fuertemente del campo científico ${ }^{41}$.

Igualmente, los datos publicados en el informe sobre las mujeres en la ciencia y la investigación She Figures $2015^{42}$ muestran que la proporción de mujeres investigadoras en la Unión Europea en 2012 todavía era solo el 33\%, estando muy infrarrepresentadas en puestos de alto nivel, con solo el 23,5\% del personal investigador de alto nivel (grado A) y el $20 \%$ de las jefaturas de las instituciones de educación superior. Igualmente, no más del $31 \%$ de las publicaciones tiene una autora responsable mujer entre 2011 y 2013, con un nivel de crecimiento anual lento. Las cifras también subrayan amplias y persistentes diferencias en toda Europa ${ }^{43}$.

39 Decisión del Consejo, de 3 de diciembre de 2013, por la que se establece el programa específico por el que se ejecuta «Horizonte 2020: Programa Marco de Investigación e Innovación (2014-2020)» y se derogan las Decisiones 2006/971/CE, 2006/972/CE, 2006/973/CE, 2006/974/CE y 2006/975/CE (2013/743/UE), DO L 347/965 de 20.12.2013, párr. 1031 .

40 Un análisis detallado sobre cómo se promueve la igualdad de género a lo largo de todo el ciclo en Horizonte 2020 está disponible en el Vademécum sobre igualdad de género en Horizonte 2020 (Comisión Europea, 2016b).

41 Comisión Europea (2012a).

42 Desde 2003 la Comisión Europea viene publicando trienalmente informes con estadísticas e indicadores sobre la situación de las mujeres en la ciencia y la investigación bajo la serie intitulada She Figures, el último de los cuales publicado hasta ahora corresponde a 2018 (Comisión Europea, 2019c).

43 Comisión Europea (2016a). 
Por esto, no sorprende que ese mismo año el Parlamento Europeo adopte la Resolución, de 9 de septiembre de 2015, sobre «Las carreras profesionales de las mujeres en los ámbitos científico y académico y los techos de cristal existentes» (2014/2251(INI)), en la que se insta a la Comisión y a los Estados miembros a adoptar diversas medidas y acciones positivas para garantizar la igualdad de género en los puestos académicos, el equilibrio entre la vida profesional y la vida personal y cambios institucionales que coadyuven a superar los obstáculos que impiden alcanzar la igualdad de género en estos ámbitos ${ }^{44}$. Y un año más tarde, el Instituto Europeo de Igualdad de Género publica sendos estudios sobre la promoción e integración de la igualdad de género en las instituciones académicas y organizaciones de investigación ${ }^{45}$.

\section{EVALUACIÓN DE LA INCORPORACIÓN DE LA PERSPECTIVA DE GÉNERO EN HORIZONTE 2020}

En cumplimiento de lo establecido en el art. 32 del Reglamento Marco de Horizonte 2020, el Grupo Asesor sobre Género (Advisory Group for Gender, AGG por sus siglas en inglés) ${ }^{46}$ de la Comisión Europea hizo público a finales de 2017 un informe relativo a la Evaluación intermedia de la igualdad de género como tema transversal en Horizonte 2020, que pretende identificar posibles mejoras en la implementación de la igualdad de género, ayudando a la Comisión Europea a evaluar la perspectiva de género en investigación e innovación como un asunto transversal en las diversas etapas de la implementación de Horizonte 2020, desde la definición del programa de trabajo hasta los proyectos financiados. Su objetivo es proporcionar una base sólida de pruebas para diseñar actividades e iniciativas futuras, en particular de cara a la preparación de la evaluación de impacto del próximo programa marco para investigación e innovación, solicitándose específicamente al AGG que evaluara: la relevancia de los objetivos específicos de igualdad de género de Horizonte 2020; la efectividad de las disposiciones de igualdad de género para

44 Disponible en: https://cutt.ly/5bO6Gyh.

45 European Institute for Gender Equality (2016b, 2016c).

46 El mandato del Grupo Asesor sobre Género es proporcionar asesoramiento a otros grupos asesores y a la Comisión Europea sobre la integración de la dimensión de género en el contenido de la investigación relacionada con todas las actividades en las que sea relevante, así como sus posibles interacciones con otros temas transversales, habiendo elaborado, como parte de su asesoramiento, varios documentos sobre la integración de la dimensión de género para que sirvan de referencia durante la preparación de los programas de trabajo Horizonte 2020 (Advisory Group for Gender, 2016, 2015). 
alcanzar los objetivos específicos y el progreso hacia los impactos esperados; la eficiencia de los recursos utilizados en relación con los productos, resultados e impactos específicos generados; la coherencia del enfoque de igualdad de género en Horizonte 2020 con las políticas e instrumentos clave de la UE e internacionales, así como el valor añadido para la UE de abordar cuestiones de igualdad de género en Horizonte $2020^{47}$.

El Grupo Asesor sobre Género ha llevado a cabo la evaluación de la igualdad de género de Horizonte 2020 como un asunto transversal, atendiendo a los cuatro indicadores clave de rendimiento (ICR) desarrollados de acuerdo con los requisitos legales de Horizonte 2020: ICR 1: porcentaje de mujeres participantes en los proyectos de Horizonte 2020 (fuerza de trabajo total); ICR 2: porcentaje de mujeres coordinadoras en los proyectos de Horizonte 2020; ICR 3: porcentaje de mujeres en grupos de asesoramiento, grupos de personal experto, paneles de evaluación, etc.; ICR 4: porcentaje de proyectos que tienen en cuenta la dimensión de género en el contenido de la investigación y la innovación ${ }^{48}$. En el Informe, cuyo período de evaluación abarca desde el 1 de enero de 2014 hasta el 31 de octubre de 2016 para los proyectos, y hasta el 1 de enero de 2017 para los indicadores, el AGG cuestiona que los tres objetivos establecidos por Horizonte 2020 no tengan plenamente en cuenta los conocimientos y análisis de género en ciencia e investigación acumulados en los últimos años, que revelan la naturaleza estructural y sistémica de la desigualdad de género, la existencia de una combinación compleja de sesgos horizontales (campos) y verticales (niveles) de género, y la complejidad de implementar la igualdad de género en las organizaciones de investigación y contribuir a los cambios estructurales en las mismas. En este sentido, el AGG plantea que se podría evaluar si la lógica de intervención en Horizonte 2020 tiene en cuenta los cinco problemas estructurales identificados para enfrentar un cambio de género estructural real en las instituciones de investigación presentado en el estudio realizado sobre Cambio estructural en las instituciones de investigación: mejora de la excelencia, igualdad de género y eficiencia en investigación e innovación, a saber: a) opacidad en los procesos de toma de decisiones; b) prácticas institucionales en las que los sesgos ${ }^{49}$ cognitivos

\footnotetext{
Advisory Group for Gender (2017).

Comisión Europea (2015).

49 El sesgo de género sigue siendo una causa importante de una evaluación menos favorable de las capacidades de las mujeres en investigación e innovación y uno de los mecanismos discriminatorios más difíciles de abordar. El sesgo implícito de género, como los estereotipos de género en la ciencia (asociar la ciencia con hombres más que con mujeres), todavía está presente en todos los Estados de la Unión Europea, incluso en países con altos niveles de igualdad de género, afectando el desarrollo de
} 
inconscientes operan al evaluar el mérito, la idoneidad para el liderazgo o la evaluación del desempeño; c) sesgos inconscientes de género en la evaluación de la excelencia y el proceso de revisión por pares; d) sesgo de género presentes en el contenido de la ciencia misma, y e) una organización laboral con sesgo de género que afecta a las instituciones de investigación, lo que resulta en una brecha salarial de género, acoso y concentración de poder, así como en la necesidad de reconocer la importancia del trabajo y la conciliación de la vida personal y familiar ${ }^{50}$. Sin embargo, a juicio del AGG, en una evaluación general, a excepción del ICR 4, los problemas estructurales identificados en las instituciones no se tienen plenamente en cuenta en los objetivos definidos en Horizonte $2020^{51}$.

Por tanto, en las conclusiones del AGG se considera muy positivo el hecho de que las acciones a favor de la igualdad de género se integren a lo largo del proceso de financiación de Horizonte 2020, habiéndose alcanzado algunos logros relevantes, especialmente la integración de la perspectiva de género en todo el ciclo, tanto en las disposiciones legales como en la redacción y finalización de los programas de trabajo y en las propuestas, así como en las fases de concesión y seguimiento del proyecto y de presentación de informes. Asimismo, el equilibrio de género en la toma de decisiones está cerca de lograrse, alcanzando más del $50 \%$ en grupos de asesores y el 36,7 \% en paneles de evaluación. Sin embargo, el AGG observa grandes dificultades en la recopilación de datos para su seguimiento y en la evaluación del proyecto, mejorando muy lentamente el equilibrio de género en los equipos de investigación. Por otro lado, la lógica de intervención de la evaluación del género como un tema transversal en Horizonte 2020 no desarrolla en todo su potencial el objetivo del cambio institucional, que constituye el principal impulsor del impacto

la carrera académica de las mujeres investigadoras (League of European Research Universities [LERU], 2018; Comisión Europea, 2017b).

50 Comisión Europea (2012b: 30-39).

51 Según se detalla en el Informe, aunque se alienta a las organizaciones de investigación a implementar cambios institucionales, en particular a través de planes de igualdad de género (PIG), que son estrategias integrales que incluyen una auditoría de género de la organización, medidas y objetivos para abordar las desigualdades y la supervisión del progreso alcanzado, y aunque Horizonte 2020 proporciona fondos a las organizaciones de investigación para los PIG en uno de los temas dentro del programa de trabajo "Ciencia con y para la Sociedad», sin embargo, la financiación es muy baja en términos absolutos, pues para el tema «Apoyo a las organizaciones de investigación para implementar planes de igualdad de género» el presupuesto fue de catorce millones para el Programa de Trabajo 2014-2015 y aún menor (diez millones) para el de 2016-2017 (Advisory Group for Gender, 2017: 7-8). 
para una mayor igualdad en investigación e innovación. De igual manera, considera el Grupo Asesor que aunque el equilibrio de género en la toma de decisiones es un objetivo importante per se porque apoya a las mujeres participantes en sus carreras, sin embargo, no puede suponerse que un mayor número de mujeres en paneles o grupos de personal experto conducirá a una selección más equitativa y justa y/o a una ciencia más sensible al género, de ahí que la experiencia en género también deba ser un criterio para la composición de grupos o paneles. Finalmente, hasta la fecha del informe muy pocos proyectos implementan realmente un análisis exhaustivo de sexo/género que desarrolle una perspectiva de género en el contenido de la investigación y el diseño del proyecto y rara vez aplican la capacitación en género dentro de ellos ${ }^{52}$.

Por todo, el AGG ofrece una serie de recomendaciones y propone introducir mejoras en la implementación de la igualdad de género en Horizonte 2020 en varios niveles. Así, el avance del conocimiento de género para analizar dónde están las desigualdades y cómo enfrentarlas, así como para comprender los obstáculos a una integración adecuada del género en el contenido de la investigación, debe ser un cuarto objetivo de la política estratégica para la igualdad de género como tema transversal, debiendo hacerse visible en todas las áreas de Horizonte 2020 y perseguirse y valorarse claramente. Asimismo, la forma en que el género interactúa con otros ejes (edad, clase, origen geográfico o étnico, etc.) de desigualdad (interseccionalidad) debe ser también un tema principal. Igualmente, mejorar la integración de los resultados de la investigación realizada en el área "Ciencia con y para la sociedad" sobre cuestiones de género en carreras, evaluación y dimensión de género de la investigación (es decir, el enfoque de cambio institucional/estructural, los mecanismos de sesgos inconscientes, los obstáculos para lograr el equilibrio de género en los equipos de investigación) permitiría que la política de género evolucionara y se focalizara de manera más precisa. Por lo que se refiere a la mejora del seguimiento y recopilación de datos, el AGG recomienda el desarrollo de indicadores más relevantes para supervisar la implementación de la igualdad de género en las diferentes partes de Horizonte 2020, siendo especialmente necesario mejorar el marcado de temas con contenido de género, de manera que una simple búsqueda de palabras permita una identificación más precisa de estos temas ${ }^{53}$. También se debe proporcionar una demostración completa

\section{Ibid: 34 .}

53 En relación con el ICR 4 (porcenaje de proyectos con una dimensión de género en contenido de investigación e innovación), la noción de una dimensión de género en los contenidos de investigación e innovación es compleja y difícil de reducir a un 
sobre cómo se debe integrar la dimensión de género en el contenido de la investigación, proponiéndose igualmente mejorar la implementación de la perspectiva de género en los todos los niveles del procedimiento, tanto en el programa de trabajo, como en los temas, propuestas, evaluación, acuerdos y convenios, proyectos, informes, etc. ${ }^{54}$

Finalmente, se ofrecen una serie de recomendaciones en relación con cada uno de los tres objetivos establecidos. Así, por lo que se refiere al equilibrio de género en los equipos de investigación, en el Informe se propone que se pida a las personas solicitantes que proporcionen una tabla resumen con indicaciones de género y posiciones dentro del proyecto; se supervise el equilibrio de género en posiciones de alto nivel dentro de los proyectos, y se definan por la Comisión objetivos específicos de equilibrio de género por sector/disciplina. En relación con el equilibrio de género en la toma de decisiones, en el Informe se recomienda que se alcance rápidamente el objetivo del $40 \%$ del sexo infrarrepresentado en grupos de personal experto; se revisen los criterios de evaluación y la forma en que se aplican, eliminándose los sesgos de género implícitos en la evaluación de los logros de investigación de mujeres y hombres, y se garantice la existencia de personas expertas con experiencia probada en género en grupos de personal experto, paneles de evaluación y grupos asesores de los diferentes campos científicos. Y en cuanto a la integración de la dimensión de género en el contenido de la investigación e innovación, en el Informe se insta a que se realicen más esfuerzos para mejorar la comprensión de la noción de dimensión de género en el contenido de investigación e innovación a nivel del personal solicitante, evaluador y entre el personal de la Comisión y la agencia; se explique cómo debe incluirse la dimensión de género en los temas y niveles; se desarrollen pautas simples para la inclusión y evaluación de la dimensión de género en el contenido de la investigación, y se capacite a miembros de los paneles de evaluación y coordinación sobre cómo considerar la dimensión de género, evitando sesgos inconscientes ${ }^{55}$.

Un año más tarde, en el informe elaborado por el Grupo de Trabajo Permanente sobre Género en la Investigación y la Innovación, órgano asesor del Comité para el Espacio Europeo de Investigación e Innovación (ERAC por

solo indicador, aunque como técnica de identificación rápida se podría hacer una búsqueda de palabras como género, sexo, mujeres, de manera que cuando un proyecto integra completamente una dimensión de género estas palabras aparecerán en varios puntos del proyecto, como los objetivos, los antecedentes teóricos, la metodología, el impacto y la difusión (Advisory Group for Gender, 2017: 35).

55 Ibid.: 37-38. 
sus siglas en inglés) del Consejo, relativo a La implementación de las Conclusiones del Consejo de 1 de diciembre de 2015 sobre el avance de la igualdad de género en el ERA, publicado en noviembre de 2018, se constata que muchas de las acciones y medidas establecidas en las Conclusiones del Consejo no han sido abordadas por la mayor parte de los países, siendo considerada la igualdad de género en algunos un problema relacionado solo con la representación de las mujeres en la investigación en lugar de considerarlo una combinación compleja de los tres objetivos relativos al equilibrio de género en los equipos de investigación y la toma de decisiones y en relación con la integración de la dimensión de género en la investigación, de ahí que se recomiende: acelerar las acciones para promover la igualdad de género, especialmente en los países de la Unión que han avanzado menos en este ámbito; mejorar la efectividad de las políticas implementadas en los países más activos, mediante sistemas de seguimiento y evaluación sólidos; definir indicadores concretos para la prioridad de igualdad de género, así como aumentar el presupuesto y considerar incentivos para que los países de la UE que menos han avanzado en la materia implementen la igualdad de género en los marcos nacionales de investigación e innovación ${ }^{56}$.

Por otro lado, los datos publicados en She Figures 2018 muestran que el desequilibrio de género entre el personal investigador sigue siendo el mismo que en 2015, pues solo un tercio son mujeres. Asimismo, la precariedad en las condiciones de trabajo afecta mayormente a las mujeres investigadoras, que representan el $13 \%$ del trabajo a tiempo parcial, frente al $8 \%$ de los hombres. Igualmente se mantiene una considerable brecha salarial de género, ya que en toda la UE las mujeres ganaron un promedio de un $17 \%$ menos que los varones en 2014, brecha salarial que se amplía con la edad. Y por lo que se refiere a la presencia de las mujeres en los puestos de alto nivel, persiste su infrarrepresentación, ya que la proporción de mujeres entre las jefaturas de instituciones de educación superior es del $22 \%$ en $2017^{57}$.

Por tanto, no extraña que en el Informe de progreso del ERA de 2018, publicado a principios de 2019, y que evalúa los avances alcanzados en la implementación de los seis objetivos prioritarios definidos para el ERA durante 2016-2018, en relación con la prioridad 4, relativa a «la igualdad de género y la integración de la dimensión de género en la investigación», se afirme expresamente que aunque la mayoría de los países han prosperado en el establecimiento de estrategias más integrales para avanzar en la consecución de la igualdad de género, sin embargo, el progreso es lento y desigual entre

56 Standing Working Group on Gender in Research and Innovation (2018).

57 Comisión Europea (2019c). 
los distintos países, de ahí que los esfuerzos para aumentar la incorporación y continuidad de las mujeres en la ciencia, implementar políticas de equilibrio entre la vida personal y profesional, reducir la brecha salarial entre mujeres y hombres y eliminar los obstáculos que impiden la promoción de la carrera de las mujeres, así como integrar mejor la dimensión de género en el contenido de investigación, sigan siendo necesarios para lograr la igualdad de mujeres y hombres en el ERA ${ }^{58}$.

Para que efectivamente se alcancen tales objetivos, es necesario que el nuevo Programa Marco incluya las recomendaciones realizadas por el Grupo Asesor sobre Género en la Evaluación intermedia de la igualdad de género como tema transversal en Horizonte 2020, analizadas con detenimiento más arriba $^{59}$, así como las recomendaciones contenidas en la Orientación para facilitar la implementación de los objetivos para promover la igualdad de género en investigación e innovación, publicada en 2018 por el Grupo de Trabajo Permanente sobre Género en la Investigación y la Innovación del ERAC, especialmente: recopilar y publicar datos desagregados por sexo relativos al desempeño de cátedras y posiciones de gestión y liderazgo; promover el equilibrio de género en los puestos de decisión y profesorado; institucionalizar los planes de igualdad de género como una herramienta de evaluación y hacerlos obligatorios para las universidades y las organizaciones de investigación; institucionalizar la proporción de mujeres en cátedras como criterio de evaluación (acreditación de educación superior, contratos programa con universidades); establecer e implementar objetivos y/o cuotas a través de la legislación; evaluar regularmente la implementación de cuotas y/o metas, e introducir incentivos para las instituciones que adopten medidas proactivas y/o sanciones por incumplimiento, en su caso ${ }^{60}$. Deben incluirse, igualmente, las recomendaciones contenidas en el informe publicado recientemente en 2020 por el Grupo de Expertos H2020 sobre Innovaciones de Género de la Comisión Europea, que incluye herramientas metodológicas para el análisis de sexo/género y propuestas para la integración de la dimensión de género en los programas de trabajo, áreas claves de investigación e innovación, clústeres y misiones del próximo Programa Marco Horizonte Europa ${ }^{61}$.

58 Comisión Europea (2019a: 9-10). En el informe técnico que acompaña al Informe de Progreso del ERA de 2018, donde se incluye la recopilación y desglose de datos, se analizan detenidamente los distintos aspectos incluidos en la prioridad 4 (Comisión Europea, 2019b: 63-76).

59 Advisory Group for Gender (2017).

60 Comisión Europea (2018: 4).

61 Comisión Europea (2020: 36-51). 


\section{HACIA LA CONSECUCIÓN DE LA IGUALDAD DE MUJERES Y HOMBRES Y LA INTEGRACIÓN DE LA DIMENSIÓN DE GÉNERO EN LA INVESTIGACIÓN EN EL PROGRAMA MARCO HORIZONTE EUROPA (2021-2027)}

Atendiendo a los limitados resultados que reflejan los estudios de evaluación y seguimiento de Horizonte 2020, no sorprende que la «Propuesta de la Comisión Europea, de 7 de junio de 2018, de Reglamento del Parlamento Europeo y del Consejo por el que se crea el Programa Marco de Investigación e Innovación Horizonte Europa y se establecen sus normas de participación y difusión para el período $2021-2017{ }^{62}$, instituya la igualdad de género como un principio transversal, señalando expresamente que las actividades desarrolladas en el marco del Programa deben dirigirse a eliminar las desigualdades de género y a promover la igualdad de mujeres y hombres en materia de investigación e innovación, de conformidad con lo establecido en los arts. 2 y 3 del Tratado de la Unión Europea y el art. 8 TFUE, debiendo integrarse la dimensión de género (análisis de sexo/género) adecuadamente en el contenido de la investigación e innovación e implementarse en todas las etapas del ciclo de la investigación (preámbulo, párr. 28), prestándose especial atención en garantizar el equilibrio entre los sexos en los paneles de evaluación y en los órganos y grupos de personal experto, según lo permita la situación del ámbito de investigación e innovación en cuestión (art. 6.9) ${ }^{63}$.

Asimismo, en la «Propuesta de la Comisión Europea, de 7 de junio de 2018, de Decisión del Parlamento Europeo y del Consejo que establece el Programa Específico por el que se ejecuta el Programa Marco de Investigación e Innovación Horizonte Europa», se señala expresamente que la planificación estratégica del Programa impulsará la promoción efectiva de la igualdad entre hombres y mujeres, incluida la integración de la dimensión de género en el contenido de la investigación y la innovación. Igualmente, en el clúster 2, relativo a "una sociedad inclusiva y segura» del pilar II, centrado

62 La propuesta del Programa Marco de Investigación e Innovación de la Unión Europea Horizonte Europa (2021-2027) se plantea en términos de continuidad del Programa Horizonte 2020, manteniéndose la estructura de los tres pilares, aunque se incluye un nuevo planteamiento intersectorial basado en clústeres (pilar 2), la definición de una serie de misiones específicas dirigidas a relacionar mejor la investigación e innovación de la UE con las necesidades de la sociedad y la ciudadanía, con una gran visibilidad e impacto, así como el Consejo Europeo de Innovación como ventanilla para ayudar a las empresas pequeñas a innovar y expandirse. Véase la información disponible en: https://bit.ly/3voZGxL.

COM (2018) 435 final. Disponible en https://cutt.ly/BbPrdnr. 
en «desafíos mundiales y competitividad industrial», se incluye expresamente en las líneas generales del ámbito de intervención referido a «democracia» las consecuencias de las desigualdades económicas y sociales sobre la participación política y las democracias, demostrándose la forma en que la corrección de las desigualdades y la lucha contra toda forma de discriminación, incluida la discriminación de género, pueden sustentar la democracia. De igual manera, en las líneas generales del ámbito de actuación relativo a «transformaciones sociales y económicas" se incluyen los nuevos tipos de trabajo, las tendencias y cambios en los mercados laborales y en las rentas del trabajo en las sociedades contemporáneas, así como sus efectos sobre la distribución de la renta, la no discriminación de género y la inclusión social. Y en las líneas generales del subprograma centrado en «reformar y mejorar el sistema de investigación e innovación de la UE» del programa transversal relativo a Reforzar el Espacio Europeo de Investigación, que financia acciones de soporte para el resto de acciones de Horizonte Europa relacionadas con las prioridades políticas del desarrollo del ERA que fomenten su coherencia y cohesión, se incluye expresamente la vinculación del ERA con el Espacio Europeo de Educación Superior mediante el apoyo a la modernización de las universidades a través de incentivos que promuevan los planes de igualdad de género, el apoyo a la igualdad de género en las carreras científicas y en la adopción de decisiones, así como la integración de la dimensión de género en el contenido de las investigaciones e innovaciones ${ }^{64}$.

Tal como se ha planteado en el marco del Proyecto GENDERACTION (Gender Equality in the ERA Community to Innovate Policy Implementation $)^{65}$, para garantizar efectivamente una mayor inclusión y visibilidad de los objetivos de igualdad de género y de la integración de la dimensión de género en el contenido de la investigación e innovación previstos en la Propuesta de la Comisión Europea para el Programa Marco, es conveniente que Horizonte Europa incluya la igualdad de género como un área específica de intervención

${ }_{64}$ COM (2018) 436 final, Anexo I. Disponible en https://cutt.ly/7bPrg5I. Una evaluación detallada del impacto que está previsto alcance el nuevo Programa Horizonte Europa realizada por la Comisión, incluida la igualdad de género, está disponible en: https://bit.ly/32Zhaoc.

65 GENDERACTION (2017-2021) es un proyecto financiado en el marco de Horizonte 2020 en el que se integran representantes de trece Estados miembros y varios países asociados con el objetivo de facilitar la creación de redes para la generación de conocimiento y el desarrollo de capacidades y competencias en el ámbito de la implementación de la igualdad de género y la integración de la dimensión de género en la investigación e innovación de la prioridad 4 del ERA en los países participantes. Toda la información está disponible en: http://genderaction.eu/. 
y financiación en el clúster relativo a «sociedades inclusivas y seguras» del pilar II sobre "desafíos globales y competitividad industrial» e integre explícitamente el género como un tema transversal en todos los grupos y áreas de intervención del mismo; integre efectivamente el género como un tema transversal en el Programa Marco; refuerce el equilibrio de género y la integración de la dimensión de género en el contenido de la investigación en las normas de funcionamiento de Horizonte Europa; revise la formulación y aplicación de los criterios de evaluación desde una perspectiva de género; garantice que la experiencia en género esté incluida en grupos de personas expertas, equipos de investigación, paneles de evaluación y grupos asesores y en las negociaciones del propio Horizonte Europa, y mantenga una línea de financiación específica para cuestiones de igualdad de género en la parte relativa al «fortalecimiento del ERA ${ }^{66}$. Asimismo, para fortalecer la igualdad de género en el ERA es necesario que Horizonte Europa incluya en la parte de la propuesta relativa a «fortalecimiento del ERA» mejores estadísticas de género y estudios cualitativos desde una perspectiva de género para el seguimiento y evaluación del Programa Marco; difusión y explotación de los resultados que tenga en cuenta el lenguaje y la imagen inclusiva de género, así como los diferentes intereses, roles y actividades de mujeres y hombres; modernizar más las universidades europeas y otras organizaciones de investigación y desarrollo a través de políticas innovadoras de igualdad de género y herramientas más efectivas desarrolladas por proyectos en materia de mujeres y ciencia, y la consideración de "ciencia, sociedad y ciudadanía» como un programa de trabajo que integre el género en todas las actividades e incluya una línea de financiación específica para acciones de coordinación y apoyo para promover la igualdad de género en investigación e innovación ${ }^{67}$. Igualmente, para garantizar una integración adecuada del principio de igualdad de género en las normas de participación de Horizonte Europa es necesario desarrollar medidas especiales de carácter temporal (acción positiva) en las normas de participación para promover la participación de las mujeres en los proyectos de Horizonte

66 Genderaction (2018a). GENDERACTION ha publicado en 2018 y 2019 diversos documentos con propuestas para mejorar la implementación de la igualdad de género y la integración de la dimensión de género en el contenido de la investigación en el Programa Marco de Investigación e Innovación Horizonte Europa, especialmente en relación con los retos sobre salud y sociedades inclusivas y seguras, digital e industria, clima, energía y movilidad, alimentos y recursos naturales del segundo pilar relativo a «desafíos globales y competitividad industrial», así como en relación con la parte de la propuesta relativa a «fortalecimiento del ERA», que están disponibles en: http:// genderaction.eu/horizon-europe/.

67 Genderaction (2018b). 
Europa, especialmente el equilibrio de género de las posiciones de liderazgo de los proyectos; diseñar medidas efectivas de conciliación de la vida personal, familiar y laboral que se apliquen en los proyectos; incluir la obligación de las instituciones asociadas de garantizar la igualdad de remuneración en las propuestas presentadas para su financiación; fortalecer los requisitos y el seguimiento de la integración de la dimensión de género en las propuestas a través de una casilla de análisis de sexo/género en las solicitudes y la experiencia de género dentro del consorcio; incluir las cuestiones de género entre los criterios de evaluación (art. 25 de la Propuesta de Reglamento), para que las propuestas se evalúen sobre la base de la excelencia, incluida la integración del análisis de sexo/género cuando la investigación se refiera a seres humanos o tenga impacto sobre los mismos; garantizar el equilibrio de género en la composición de los comités de evaluación y el personal evaluador (art. 26 de la Propuesta de Reglamento), incluyéndose personal experto en género entre las personas evaluadoras y la formación en género para el personal evaluador, para combatir el sesgo de género en la evaluación ${ }^{68}$.

Posteriormente, en la Comunicación de la Comisión al Parlamento Europeo, al Consejo, al Comité Económico y Social Europeo y al Comité de las Regiones, de 30 de septiembre de 2020, relativa a «un nuevo ERA para la investigación y la innovación", se reconoce que los avances han sido lentos e insuficientes, a pesar de la atención concedida a la igualdad de género en las políticas de I+i, como reflejan los datos publicados en She Figures 2018, de ahí que el ERA deba reforzar la participación de la mujer en todos los ámbitos, especialmente en la ciencia, la tecnología, la ingeniería y las matemáticas (CTIM, STEM por sus siglas en inglés), instando la Comisión a la adopción e implementación de planes de igualdad de género inclusivos a partir de 2021, de conformidad con los objetivos de Horizonte Europa y con miras a promover la igualdad de género para fortalecer el potencial en la I+i de la UE (Acción 12) ${ }^{69}$. Igualmente, en las Conclusiones del Consejo, de 1 de diciembre de 2020, sobre el Nuevo Espacio Europeo de Investigación, se pide a la Comisión y a los Estados miembros que acuerden acciones prioritarias en el ámbito de la igualdad de género como parte de una agenda política del ERA en 2021, adoptándose un enfoque renovado en la transversalización de la igualdad de género, incluso a través de planes de igualdad de género, y la integración de la dimensión de género en el contenido de la I+i, invitándose a los Estados y a las organizaciones de financiación de la investigación a promover medidas

68 Genderaction (2018c).

69 COM (2020) 628 final. Disponible en: https://cutt.ly/HbPtcR9. 
para garantizar que la asignación de la financiación de la investigación no se vea afectada por sesgos de género (párr. 27.ii) ${ }^{70}$.

Atendiendo a las medidas planteadas en la Comunicación de la Comisión $\mathrm{y}$ a las recomendaciones formuladas por diversos grupos expertos anteriormente analizadas, se observan ciertos avances en la integración de la igualdad de género en los últimos documentos de Horizonte Europa, conforme al acuerdo parcial alcanzado por el Consejo de la Unión Europea el 15 de abril de 2019 ${ }^{71}$. Así, en la «Propuesta de Decisión del Consejo de 29 de septiembre de 2020, sobre el establecimiento del programa específico de ejecución de Horizonte Europa: el Programa Marco de Investigación e Innovación», entre los objetivos operativos se ha incluido el fortalecimiento de la dimensión de género en todo el Programa (art. 2.ca). Igualmente, el plan estratégico plurianual deberá incluir entre los temas específicos la igualdad de género, incluida la integración de la dimensión de género en el contenido de la I+i (art. 4a.3.d). Asimismo, en la presentación del pilar II, relativo a «desafíos mundiales y competitividad industrial europea», se afirma expresamente que la igualdad de género es un factor crucial para lograr un crecimiento económico sostenible, siendo importante, en

70 Consejo de la Unión Europea (2020a).

71 El 15 de abril de 2019 se acordaron partes fundamentales de la «Propuesta de Decisión del Consejo que establece el Programa Específico por el que se ejecuta el Programa Marco de Investigación e Innovación Horizonte Europa», incluyéndose las líneas generales de las actividades de investigación en cada ámbito de Horizonte Europa, el proceso de planificación estratégica plurianual, que adoptará la Comisión con la participación de los Estados miembros, la creación del Consejo Europeo de Innovación, los programas de trabajo que ejecutan el programa específico y que adoptará la Comisión, la ampliación de la participación en actividades de investigación financiadas en el marco de Horizonte Europa y el refuerzo del Espacio Europeo de Investigación. El programa se basa en tres pilares complementarios e interrelacionados: el primer pilar, relativo a «excelencia científica», apoya la excelencia en la ciencia pura, refuerza el liderazgo científico de la Unión y promueve los conocimientos y las capacidades de gran calidad; el segundo pilar, denominado «desafíos mundiales y competitividad industrial europea», apoya la investigación que aborda desafíos sociales, así como las tecnologías industriales en ámbitos como la sanidad, la seguridad, las tecnologías facilitadoras esenciales y digitales, el clima, la energía, la movilidad, la alimentación y los recursos naturales, introduciéndose, además, un número limitado de misiones de investigación que comprenderán una cartera de actividades de investigación; y el tercer pilar, referido a "Europa innovadora», se dedica a la expansión de la innovación de vanguardia y disruptiva a través de la creación del Consejo Europeo de Innovación, que será una ventanilla única para innovadores que tengan un gran potencial, véase la Propuesta de Decisión en: https://bit.ly/3aLnEvq. 
consecuencia, integrar una perspectiva de género en todos los desafíos globales que lo conforman. De igual manera, en el ámbito de actuación relativo a «transformaciones sociales y económicas» del clúster II (cultura, creatividad y sociedad inclusiva) se incluye expresamente como línea de actuación específica la adopción y desarrollo de estrategias avanzadas y métodos innovadores para la igualdad de género en todos los ámbitos sociales, económicos y culturales, así como para hacer frente a los prejuicios y la violencia de género $(2.2 .3)^{72}$.

Dos meses más tarde, el acuerdo adoptado el 11 de diciembre de $2020^{73}$, que contiene la «Propuesta de Reglamento del Parlamento Europeo y del Consejo por el que se crea Horizonte Europa: Programa Marco de Investigación e Innovación», por el que se establecen sus normas de participación y difusión, añade al párr. 28 del preámbulo el principio de igual retribución reconocido en el art. 157 TFUE. Asimismo, en el marco de los principios se señala expresamente que el Programa garantizará la promoción efectiva de la igualdad de oportunidades y la implementación de la integración de la perspectiva de género y de la dimensión de género en el contenido de la investigación y la innovación, teniendo como objetivo abordar las causas del desequilibrio de género (art. 6.a.5). Igualmente, en los criterios de adjudicación y selección se incluye expresamente el género como condición para abordar las propuestas (art. 25.3). De igual modo, en el nombramiento de las personas expertas independientes, tanto la Comisión como el organismo de financiación de que se trate deben tomar las medidas adecuadas para logar una composición equilibrada en los grupos de expertos y paneles de evaluación atendiendo a su formación y especialización en materia de género (art. 44.4). Y se publicarán y analizarán los datos relativos a la participación de género en las acciones financiadas en el proceso de supervisión de Horizonte Europa que lleve a cabo la Comisión $(\text { art. } 45.1 .1 i)^{74}$.

Tras el acuerdo alcanzado en diciembre de 2020, el primer Plan Estratégico 2021-2024, adoptado el 15 de marzo de 2021 y que establece las orientaciones estratégicas, el contenido de los programas de trabajo y la convocatorias de propuestas para los cuatro primeros años, incluye la igualdad de género entre los ocho temas horizontales, constituyendo una prioridad trans-

72 Consejo de la Unión Europea (2020b).

73 Las instituciones de la UE alcanzaron un acuerdo político sobre Horizonte Europa el 11 de diciembre de 2020 y fijaron el presupuesto en 95500 millones de euros (incluidos 5400 millones de euros de la próxima generación de la UE-Fondo de recuperación). Sobre esta base, el Parlamento Europeo y el Consejo procederán a la adopción y publicación de los actos jurídicos correspondientes. Véase la información disponible en: https://ec.europa.eu/info/horizon-europe_en.

74 Consejo de la Unión Europea (2020c). 
versal que afecta a todas las partes de Horizonte Europa, teniendo todas sus actividades el objetivo de eliminar la desigualdad de género y socioeconómicas entrecruzadas a través de los sistemas de investigación e innovación, incluso abordando los prejuicios inconscientes y las barreras estructurales sistémicas, de manera que la integración de la dimensión de género es un requisito por defecto en los contenidos de investigación e innovación en todo el Programa, salvo que su no pertinencia esté debidamente justificada, siendo esta integración particularmente relevante para los desafíos globales, especialmente en áreas como la salud y la atención, la pandemia de la COVID-19, la violencia en línea, la inteligencia artificial y la robótica o la mitigación y adaptación al cambio climático, en las que el sexo y/o las diferencias de género juegan un papel importante y, por lo tanto, determinan la relevancia social y la calidad de los resultados de la investigación y la innovación ${ }^{75}$.

Conforme al Plan Estratégico 2021-2024 de Horizonte Europa, los textos del Programa de Trabajo para 2021-2022 que se han hecho públicos hasta la fecha integran la igualdad de género como prioridad transversal a lo largo de los distintos programas y convocatorias. Así, en el Programa de Trabajo del Consejo Europeo de Investigación (European Research Council, ERC por sus siglas en inglés) para 2021, adoptado el 22 de febrero de 2021, se establece expresamente que los beneficiarios de las subvenciones del ERC deben tomar medidas para promover la igualdad de oportunidades entre hombres y mujeres en la implementación de la acción y buscar un equilibrio de género en todos los niveles del personal asignado a la acción, debiendo, asimismo, los investigadores principales determinar la relevancia de integrar el análisis de sexo/ género en su investigación, pudiendo considerarse costes subvencionables las actividades específicas orientadas a tales fines ${ }^{76}$. Igualmente, en el Programa de Trabajo del Consejo Europeo de Innovación (European Innovation Council, EIC por sus siglas en inglés) para 2021, adoptado el 17 de marzo de 2021, además de la exigencia de contar con un Plan de Igualdad de Género y de tenerse en cuenta la integración de la dimensión de género en la investigación cuando sea relevante, se establece expresamente que, para mejorar el equilibrio de género y promover mujeres innovadoras, al menos el $40 \%$ del Consejo del EIC, el Comité de Inversiones del Fondo del EIC, los jurados del EIC y los evaluadores expertos del EIC serán mujeres, con el objetivo de llegar al $50 \%$, incluyéndose también un objetivo del $40 \%$ de empresas dirigidas por mujeres ${ }^{77}$.

75 Comisión Europea (2021a: 25, 35, 36, 37, 46, 47, 48, 51, 53, 93).

76 Comisión Europea (2021b: 12).

77 Comisión Europea (2021f: 11, 38, 53, 55, 58, 67, 75, 80, 134-135). 
Por lo que se refiere al clúster 2 relativo a «cultura, creatividad y sociedad inclusiva» del pilar II, el documento provisional del Programa de Trabajo para 2021-2022 incluye una convocatoria de proyectos referida a «feminismos para una nueva era de democracia», que estarán dirigidos a examinar la contribución de los marcos teóricos modernos del pensamiento feminista y el análisis de género, incluidos, entre otros, la ética del cuidado, el ecofeminismo, la teoría interseccional y el feminismo inclusivo, la teoría queer, los estudios sobre masculinidad, así como el activismo y prácticas políticas, para la renovación de conceptos políticos fundamentales como igualdad, identidad, solidaridad, orden, seguridad, derechos individuales y colectivos, participación, diálogo, etc. en contextos democráticos modernos, así como a investigar y contrarrestar las estrategias discursivas y los efectos de las movilizaciones antigénero y antifeministas (incluyendo, entre otros, el uso de redes sociales, el discurso de odio y acoso en línea, las manifestaciones públicas y restricciones a la libertad académica) y sus conexiones con el posicionamiento de discursos populistas extremos, actores políticos y movimientos religiosos tradicionalistas. Asimismo, se incluye una convocatoria de proyectos específica en materia de «empoderamiento social, económico y cultural de género" que deberán proponer marcos teóricos que coadyuven a superar la formación de jerarquías de poder de género que conducen a formas sistemáticas y estructurales de discriminación, desigualdades sociales y económicas y a la violencia de género, abordando las interrelaciones de poder y las barreras que impiden alcanzar la igualdad de género en diferentes ámbitos sociales y económicos, especialmente los relacionados con la formulación de políticas y la toma de decisiones, la participación en el mercado laboral y la brecha salarial de género, la conciliación de las responsabilidades laborales con la esfera personal y familiar, la violencia de género, los derechos reproductivos y los roles de género en la educación y las representaciones culturales, el arte y los medios de comunicación ${ }^{78}$.

Igualmente, en el documento provisional del Programa de Trabajo para 2021-2022 correspondiente al área relativa a «reforzar el Espacio Europeo de Investigación» del programa transversal «Ampliar la participación y fortalecer el Espacio Europeo de Investigación», se contemplan varias convocatorias específicas en materia de igualdad de género, entre las que destacan especialmente la creación de un primer Centro Europeo de Excelencia sobre Igualdad de Género Inclusiva en Investigación e Innovación, destinado a promover y difundir conocimientos sobre políticas inclusivas de igualdad de género en $\mathrm{I}+\mathrm{i}$, proporcionar experiencia y apoyo a los Estados miembros, las organizaciones

78 Comisión Europea (2021c: 15-17, 87-89). 
de I+i y la Comisión Europea en el diseño, implementación y evaluación del cambio institucional a través de planes inclusivos de igualdad de género, así como proporcionar apoyo experto para la recopilación de datos paneuropeos sobre igualdad de género en $\mathrm{I}+\mathrm{i}$, debiendo incluirse información sobre la adopción e implementación de planes de igualdad de género en los Estados miembros de la UE y países asociados, y contribuir a la publicación de la serie She Figures; el desarrollo de una Red de Coordinación de representantes nacionales para avanzar en la implementación de los objetivos de igualdad e inclusión de género del ERA en los Estados miembros, y el apoyo a la implementación de planes inclusivos de igualdad de género de acuerdo con lo establecido en los criterios de elegibilidad de Horizonte Europa ${ }^{79}$.

$\mathrm{Y}$ en el documento provisional de los anexos generales del Programa de Trabajo para 2021-2022, en los que se establecen las condiciones generales aplicables a las convocatorias y temas de subvenciones y se describen los procedimientos de evaluación y adjudicación así como los parámetros para recibir financiación, se establece expresamente que a partir de 2022 para ser elegibles las entidades de los Estados miembros y países asociados que sean organismos públicos, organizaciones de investigación o establecimientos de educación superior (incluidas las organizaciones de investigación privadas y los establecimientos de educación superior) deben contar con un plan de igualdad de género que cubra los siguientes requisitos mínimos: documento formal publicado en el sitio web de la institución y firmado por la alta dirección; los recursos y la experiencia necesaria para implementarlo; los datos desglosados por sexo/género relativos al personal con informes anuales basados en indicadores; los mecanismos de sensibilización y formación sobre igualdad de género y estereotipos de género para el personal y los responsables de la toma de decisiones, así como los objetivos, medidas y acciones específicas en relación con el equilibrio entre la vida personal y laboral y la cultura organizacional; el equilibrio de género en el liderazgo y la toma de decisiones; la igualdad de género en la contratación y la progresión profesional; la integración de la dimensión de género en los contenidos de investigación y docencia y las medidas contra la violencia de género, incluido el acoso sexual. Igualmente se indica que los beneficiarios deben tener como objetivo, en la medida de lo posible, un equilibrio de género en todos los niveles del personal asignado a la acción, incluidos los niveles de supervisión y gestión ${ }^{80}$.

79 Comisión Europea (2021d: 85-86, 119-120).

80 Asimismo, entre los criterios de adjudicación establecidos para la evaluación de las distintas propuestas relativas a Acciones de Investigación e Innovación (RIA por sus siglas en inglés) y Acciones de Innovación (IA por sus siglas en inglés), así como en 


\section{CONCLUSIONES: LA NECESARIA ADOPCIÓN DE UNA DIRECTIVA RELATIVA A IGUALDAD DE MUJERES Y HOMBRES E INTEGRACIÓN DE LA PERSPECTIVA Y DIMENSIÓN DE GÉNERO EN EL ERA}

Sin duda, Horizonte Europa ha mejorado y ampliado las disposiciones relativas a igualdad de género e integración de la dimensión de género en la investigación e innovación, suponiendo un avance importante para la superación de las desigualdades de género en el ERA, al implementar la igualdad de género como un principio transversal que se proyecta sobre todo el Programa, incorporando la integración de la dimensión de género y el análisis de sexo/género en el contenido de la investigación e innovación como un requisito predeterminado de las propuestas y proyectos, salvo que su no pertinencia esté debidamente justificada, siendo esta integración particularmente relevante para los desafíos globales, especialmente en áreas como la salud y la atención, la pandemia de la COVID-19, la violencia en línea, la inteligencia artificial y la robótica o la mitigación y adaptación al cambio climático, en las que el sexo y/o las diferencias de género juegan un papel importante y, por lo tanto, determinan la relevancia social y la calidad de los resultados de la investigación y la innovación. Asimismo, se contemplan convocatorias de proyectos específicos dirigidos a examinar la contribución de los marcos teóricos modernos del pensamiento feminista (incluidos, entre otros, la ética del cuidado, el ecofeminismo, la teoría interseccional y el feminismo inclusivo, la teoría queer, los estudios sobre masculinidad, el activismo y prácticas políticas), a contrarrestar las estrategias discursivas y los efectos de las movilizaciones antigénero y antifeministas mediante el uso de redes sociales, el discurso de odio y acoso en línea, y a proponer marcos teóricos que coadyuven a superar la formación de jerarquías de poder de género que conducen a formas sistemáticas y estructurales de discriminación en diferentes ámbitos sociales y económicos, especialmente los relacionados con la formulación de políticas y la toma de decisiones, la participación en el mercado laboral y la brecha salarial de género, la conciliación de las

las acciones de cofinanciación del Programa (CoFund) se incluye expresamente la consideración adecuada de la dimensión de género en el contenido de la investigación e innovación. Igualmente, en el procedimiento de evaluación se contempla que para la selección de las propuestas con la misma puntuación dentro de una sola dotación presupuestaria se utilizará, en caso de ser necesario, como factor de priorización el equilibrio de género entre el personal responsable de la realización de las actividades de investigación y/o innovación que estén incluidos en la tabla de investigadores de la propuesta (Comisión Europea 2021e: 12-13, 18, 19, 23). 
responsabilidades laborales con la esfera personal y familiar, la violencia de género, los derechos reproductivos y los roles de género en la educación y las representaciones culturales, el arte y los medios de comunicación. Igualmente, se establece por primera vez como criterio de elegibilidad para acceder a la financiación que los organismos públicos, las organizaciones de investigación y los establecimientos de educación superior cuenten con un plan de igualdad de género inclusivo, se procede a la creación de un primer Centro Europeo de Excelencia sobre Igualdad de Género Inclusiva en Investigación e Innovación y de una Red de Coordinación de representantes nacionales para avanzar en la implementación de los objetivos de igualdad e inclusión de género del ERA en los Estados miembros.

Las diversas medidas y acciones incorporadas en el Programa Marco Horizonte Europa contribuirán directamente al objetivo de desarrollo sostenible (ODS) 5 de Naciones Unidas sobre igualdad de género, al empoderamiento de las mujeres y, en consecuencia, a mejorar el sistema europeo de investigación e innovación ${ }^{81}$. En efecto, la participación de las mujeres en la ciencia y la tecnología coadyuva a aumentar la calidad, la relevancia social y la competitividad de la investigación y la innovación. Específicamente, el equilibrio de género en la toma de decisiones contribuye a avanzar en la consecución de la igualdad de oportunidades de mujeres y hombres y a ampliar el alcance de las políticas de investigación e innovación y, por lo tanto, a atender mejor las necesidades de todos los sectores de la sociedad ${ }^{82}$. Asimismo, la integración de la dimensión de género contribuye a la excelencia investigadora, viéndose afectada la calidad de la investigación cuando la dimensión de género no se tiene en cuenta, pues el género es una variable analítica y explicativa clave en la investigación, al ser el género resultado de un proceso sociocultural que se articula en torno a los valores culturales y las actitudes sociales que, en conjunto, dan forma y sancionan los comportamientos de mujeres y hombres, afectando a los productos, las tecnologías, los entornos y el conocimiento, coadyuvando la integración de la dimensión de género a repensar los estándares y modelos científicos de referencia que discriminan a las mujeres. Por tanto, la integración de la dimensión de

81 En la Agenda 2030 para el Desarrollo Sostenible, adoptada por la Asamblea General el 25 de septiembre de 2015, que consta de 17 objetivos de desarrollo sostenible (ODS) y 169 metas, se incluye el ODS 5 relativo a lograr la igualdad de género y empoderar a todas las mujeres y niñas. Véase la Resolución de la Asamblea General 70/1, de 25 de septiembre de 2015, relativa a "Transformar nuestro mundo: la Agenda 2030 para el Desarrollo Sostenible». Disponible en https://bit.ly/3u5Z3t2.

82 Comisión Europea (2018). 
género coadyuva a cuestionar las normas y estereotipos de género, repensar los estándares y modelos de referencia y comprender las diversas necesidades de género, contribuyendo, en consecuencia, a la excelencia investigadora e incrementando la relevancia social del conocimiento y de las tecnologías e innovaciones, a cuya consecución se dirige el ERA ${ }^{83}$.

Con todo, hay que considerar el limitado alcance de las medidas adoptadas para garantizar la efectiva consecución de la igualdad en el ámbito de la investigación e innovación al seguir afectada por un conjunto de desigualdades de género estructurales y persistentes, especialmente la segregación de género en investigación y ciencia, los retos en la carrera profesional relacionados con el género, el desequilibrio de género en cargos superiores en la academia, los sesgos de género en el acceso a la financiación de la investigación, la investigación ciega al género y sesgada en el género y la cultura organizativa y el proceso institucional ciego al género y con sesgo de género ${ }^{84}$, que difícilmente pueden erradicar medidas como la adopción de planes de igualdad de género que responden frecuentemente a una mera formalidad cuya efectiva implementación y eficacia está condicionada, en la mayoría de los Estados miembros, por la falta de recursos, financiación y dotación presupuestaria, así como por carecer de efectivos mecanismos de auditoría y rendición de cuentas en caso de incumplimiento (Drew et al., 2017). Sin olvidar que la simple incorporación del $40 \%$ de mujeres a los puestos de toma de decisiones no es suficiente si el único objetivo es cumplir con los requisitos formales de participación equilibrada de género, ni tampoco sería admisible la introducción del análisis de sexo/género solo cosméticamente, de manera que solo se incluyera la variable sexo, pero no la perspectiva de género en el análisis (Sánchez et al., 2017).

Asimismo, la efectiva implementación de las diversas medidas y acciones dirigidas a la consecución de la igualdad de género en los próximos siete años de vigencia del Programa Marco Horizonte Europa va a estar notablemente limitada por las resistencias persistentes individuales e institucionales interconectadas explícitas e implícitas que se han constatado cuando se implementan políticas de igualdad de género en investigación e innovación, como reflejan los diversos obstáculos que provocaron una aplicación ineficaz de la transversalización de género en el Sexto Programa Marco (2002-2006), el Séptimo Programa Marco (2007-2013) así como en Horizonte 2020, revelándose tensiones entre el mandato de la Unión Europea y su efectiva implementación. Como han demostrado diversos estudios, los principales

83 Comisión Europea (2020: 8).

84 European Institute for Gender Equality (2016d). 
actores de las estructuras relacionales formales e informales de las instituciones de investigación se resisten, reproduciendo y manteniendo las desigualdades de género, diluyéndose, por tanto, la virtualidad transformadora de los cambios organizacionales favorables a la igualdad de mujeres y hombres que persigue la integración transversal de la perspectiva de género en la investigación e innovación (Vida, 2020; Mergaert y Lombardo, 2014).

Igualmente, en los marcos jurídicos nacionales no existe una regulación homogénea en esta materia, careciendo el marco normativo de la mayoría de los Estados miembros de incentivos, cuotas e indicadores eficaces y obligatorios que coadyuven a la igualdad de género en el campo de la investigación y la innovación. Y aunque en algunos países se contempla una obligación general de las organizaciones públicas de investigación de dotarse de órganos que promuevan la igualdad y planes de acción, sin embargo no se proporciona la financiación suficiente ni se prevén mecanismos de medición del impacto, rendición de cuentas ni sanciones cuando no se alcanzan los objetivos establecidos. De ahí que, a pesar de haber transcurrido ya más de veinte años desde la publicación del Informe ETAN, los progresos han sido lentos e insuficientes (Comisión Europea, 2019a) porque no es fácil generar un cambio cultural y organizacional favorable a la igualdad de mujeres y hombres mediante el desarrollo de estrategias de implementación que coadyuven a superar el sesgo de género en todos los niveles de la toma de decisiones si éstas están reguladas en disposiciones normativas que tienen carácter dispositivo, carecen de fuerza vinculante y no imponen sanciones cuando no se respetan.

Por todo, para superar el nivel meramente institucional (Leisyte, 2019), asegurar la efectiva implementación de las diversas medidas y acciones previstas en los próximos siete años de vigencia del Programa Marco Horizonte Europa, garantizar la armonización normativa y brindar una igual protección, oportunidades y derechos en el espacio común, es indispensable que la Unión Europea adopte un acto legislativo vinculante en forma de directiva en materia de igualdad de mujeres y hombres en investigación e innovación que obligue a todos los Estados miembros de manera armonizada, homogénea e igual a adoptar disposiciones legislativas específicas de carácter vinculante en sus estrategias nacionales de investigación e innovación, que garanticen un sistema coherente para la recopilación de datos estadísticos así como un planteamiento homogéneo de indicadores de impacto, revisión, evaluación y rendición de cuentas por todos los Estados miembros y exijan la efectiva implementación de las recomendaciones realizadas por el Grupo de Trabajo Permanente sobre Género en la Investigación y la Innovación del ERAC sobre la prioridad de la igualdad de género en el ERA para 2020-2030, centradas en una aproximación interseccional de la igualdad de género con otros factores como la edad, el estado de salud, la discapacidad, la ocupación, el nivel 
socioeconómico, la situación migratoria o la ubicación geográfica, así como en un enfoque integrado de cambio institucional de las condiciones marco que coadyuven a la superación de los sesgos y brechas de género y la eliminación de las barreras estructurales en las carreras profesionales de las mujeres en la investigación de forma sincronizada en todos los Estados miembros, configurándose unas estructuras nacionales estables y responsables ${ }^{85}$.

Así, mediante una directiva se podrá requerir a los Estados miembros que adopten medidas homogéneas y eficaces que aborden los desequilibrios de género en las instituciones de investigación y los órganos de toma de decisiones (establecimiento, implementación y supervisión de objetivos y/o cuotas que aseguren al menos un $40 \%$ del sexo menos representado; institucionalización de al menos un $40 \%$ de mujeres en cátedras como criterio de evaluación para la acreditación de educación superior o contratos programa con universidades; implementación de medidas especiales de carácter temporal para garantizar al menos un $40 \%$ de mujeres en posiciones de liderazgo de los proyectos; revisión de los criterios de evaluación y la forma en que se aplican, eliminándose los sesgos de género implícitos en la evaluación de los logros de investigación de mujeres y hombres; diseño de medidas efectivas de conciliación de la vida personal y profesional que se apliquen en los proyectos, y reducción de la brecha salarial de género, garantizándose la igualdad de remuneración en las propuestas presentadas para su financiación), garanticen la efectiva integración de la dimensión de género en las políticas, programas y proyectos de investigación (fortalecimiento de los requisitos y el seguimiento de la integración de la dimensión de género en las propuestas a través del cuadro de análisis de sexo/género en las aplicaciones; inclusión de temas de investigación e innovación con responsabilidad de género entre los criterios de adjudicación; inclusión de la igualdad de género como un área específica de intervención y financiación; integración efectiva del género como un tema transversal; desarrollo de pautas para la inclusión y evaluación de la dimensión de género en el contenido de la investigación e innovación; inclusión de la experiencia en género en los grupos de personas expertas, equipos de investigación, paneles de evaluación y grupos asesores; difusión de los resultados de forma que se tenga en cuenta el lenguaje y la imagen inclusiva de género, así como los diferentes intereses, roles y actividades de mujeres y hombres), y establezcan sistemas de monitorización y rendición de cuentas que incluyan indicadores específicos de evaluación y consecución de los objetivos relacionados con la implementación de la igualdad de género y la integración del análisis de sexo/género en la investigación e innovación, introduciéndose

85 Standing Working Group on Gender in Research and Innovation (2020a). 
incentivos para las instituciones que adopten medidas proactivas y sanciones, en su caso, por incumplimiento.

La adopción de una directiva no solo garantizará la consecución del objetivo de eliminar las desigualdades de género y lograr la igualdad de mujeres y hombres en investigación e innovación previsto para los próximos siete años de vigencia del Programa Marco Horizonte Europa de forma homogénea y armonizada en todos los Estados miembros, asimismo también evitará el avance de los retrocesos que se aprecian a raíz del impacto de la pandemia de la COVID-19, como se expone en el «Documento de posición sobre el brote actual de COVID-19 y los impactos de género en investigadores y docentes», publicado por el Grupo de Trabajo Permanente sobre Género en la Investigación e Innovación del ERAC el 2 de junio de 2020, que está afectando de manera desproporcionada a la actividad y productividad de las investigadoras, especialmente de las que tienen que atender responsabilidades de cuidados, obstaculizando su promoción profesional, exacerbando las brechas de género existentes y poniendo en jaque los logros conseguidos ${ }^{86}$. De ahí que para que no sea una mera promesa (Sánchez et al., 2017: 100), es más necesario que nunca que la UE adopte un acto legislativo vinculante que garantice avanzar de forma más efectiva durante la vigencia del Programa Marco Horizonte Europa en la consecución de la igualdad de mujeres y hombres así como en la transversalización de la perspectiva de género y la integración de la dimensión de género en el ERA.

\section{Bibliografía}

Advisory Group for Gender (2015). For a better integration of the gender dimensión in the Horizon 2020 Work Programme 2016-2017. Disponible en: https://bit. ly/3dXU6g4.

Advisory Group for Gender (2016). For a better integration of the gender dimensión in the Horizon 2020 Work Programme 2018-2020. Position paper. Disponible en: https://bit.ly/32Ueqsi.

Advisory Group for Gender (2017). Interim Evaluation: Gender equality as a crosscutting issue in Horizon 2020. Report of the Expert Group on the Interim Evaluation of Gender Equality as a crosscutting issue in Horizon 2020. Luxembourg: Directorate-General for Research and Innovation. Disponible en: https://bit. ly/3dVX5pl.

Biglia, B. y Vergés-Bosch, N. (2016). Cuestionando la perspectiva de género en la investigación. REIRE: Revista d'innovació i recerca en educació, 9 (2), 12-29. Disponible en: https://bit.ly/2R4G5nD.

86 Standing Working Group on Gender in Research and Innovation (2020b). 
Comisión Europea (2000). Science policies in the European Union. Promoting excellece through mainstreaming gender equality: A report from the ETAN expert working group on women and science. Directorate-General for Research and Innovation. Disponible en: https://bit.ly/3aI80Ru.

Comisión Europea (2012a). Commission Staff Working Document. Impact Assessment. Accompanying the document Communication from the Commission to the European Parliament, the Council, the European Economic and Social Committe and the Committe of Regions. A Reinforced European Research Area Partnership for Excellence and Growth. Brussels, 17-7-2012, SWD (2012) 212 final.

Comisión Europea (2012b). Structural change in research institutions: Enhacing excellence, gender equality and efficiency in research and innonvation. Luxembourg: Publications Office of the European Union. Disponible en: https://bit.ly/3gLGWVs.

Comisión Europea (2013a). European Research Area. Progress Report 2013, accompanied by Facts and Figures 2013. Luxembourg: Directorate-General for Research and Innovation, European Research Area. Disponible en: https://bit. ly/32P7yMU.

Comisión Europea (2013b). Gendered Innovations. How Gender Analysis Contributes to Research. Luxembourg. Disponible en: https://bit.ly/3dUhfQw.

Comisión Europea (2014). European Research Area. Progress Report 2014, accompanied by Facts and Figures 2014. Luxembourg: Directorate-General for Research and Innovation, European Research Area. Disponible en: https://bit.ly/2R30uti.

Comisión Europea (2015). Horizon 2020 indicators. Assessing the results and impact of Horizon 2020. Luxembourg: Directorate-General for Research and Innovation. Disponible en: https://bit.ly/3dYz4ht.

Comisión Europea (2016a). She Figures 2015. Luxembourg: Directorate-General for Research, Directorate B. Open Innovation and Open Science, Unit B.7 Science with and for Society. Disponible en: https://bit.ly/2ScH7ib.

Comision Europea (2016b). The EU Framework Programme for Research and Innovation. Horizon 2020. Excellent Science, Competitive Industries, Better Society. Vademecum on Gender Equality in Horizon 2020, RTD-B7 Science with and for Society. Disponible en: https://bit.ly/3t83x0Z.

Comisión Europea (2017a). Era Progress Report 2016. Report from the Commission to the Council and the European Parliament. The European Research Area: Time for Implementation and monitoring progres, COM (2017) 35, Brussels. Disponible en: https://bit.ly/3xq5OI1.

Comisión Europea (2017b). Implicit gender biases during evaluations: How to raise awareness and change attitudes? Workshop Report. Brussels, 30-31 May 2017. Disponible en: https://bit.ly/3gHdvUp.

Comisión Europea (2018). Guidance to facilitate the implementation of targets to promote gender equality in research and innovation. Prepared by the European Commission and the Helsinki Group on Gender in Research and Innovation, in consultation with the European Research Area stakeolders' plaftorm. 
Luxembourg: Directorate-General for Research and Innovation. Disponible en: https://bit.ly/3dTxsVX.

Comisión Europea (2019a). Era Progress Report 2018. Report from the Commission to the Council and The European Parliament. The European Research Area: Advancing together the Europe of research and innovation, COM (2019) 83. Luxembourg: Directorate-General for Research and Innovation. Disponible en: https://bit.ly/3dZ7kcl.

Comisión Europea (2019b). Era Progress Report 2018. Data gathering information for the 2018 ERA monitoring. Technical Report. Written by PPMI and Science-Metriz. Luxembourg: Directorate-General for Research and Innovation. Disponible en: https://bit.ly/3sVlaRp.

Comisión Europea (2019c). She Figures 2018. Luxembourg: Directorate-General for Research and Innovation. Disponible en: https://bit.ly/3aKJLCh.

Comisión Europea (2020). Gendered Innovations 2: How inclusive Analysis Contributes to Research and Innovation. Brussels: Directorate-General for Research and Innovation. Disponible en: https://bit.ly/3dUd7ju.

Comisión Europea (2021a). Horizon Europe Strategic Plan (2021-2024). Brussels: Directorate-General for Research and Innovation. Disponible en: https://bit. ly/3sZEOeX.

Comisión Europea (2021b). ERC Work Programme 2021. Disponible en: https://bit. ly/3xEllij.

Comisión Europea (2021f). EIC Work Programme 2021. Disponible en: https://bit. ly/2QDgcLZ.

Comisión Europea (2021c). Horizon Europe. Work Programme 2021-2022. 5. Culture, creativivy and inclusive society. Disponible en: https://bit.ly/2R2IMpW.

Comisión Europea (2021d). Horizon Europe. Work Programme 2021-2022. 11. Widening participation and strengthening the European Research Area. Disponible en: https://bit.ly/3xy7i2O.

Comisión Europea (2021e). Horizon Europe. Work Programme 2021-2022. 13. General Annexes. Disponible en: https://bit.ly/3eBBe5D.

Consejo de la Unión Europea (2014). Conclusion on progress in the European Research Area. Competitiveness Council Meeting. Brussels, 21 February 2014. Disponible en: https://bit.ly/32RyTOt.

Consejo de la Unión Europea (2015a). Council conclusions on advancing gender equality in the European Research Area. Brussels, 1 december 2015. Disponible en: https://bit.ly/3tW2SRt.

Consejo de la Unión Europea (2015b). Council Conclusions on the European Research Area Roadmap 2015-2020. Brussels, 19 May 2015. Disponible en: https://bit. ly/3xvyvDl.

Consejo de la Unión Europea (2020a). Council conclusiones on the New European Research Area. Brussels, 1 December 2020. Disponible en: https://bit. ly/3gLr3Ow.

Consejo de la Unión Europea (2020b). Proposal for a Decision of the Council on establishing the specific programme implementing Horizon Europe, the Framework 
Programme for Research and Innovation. Brussels, 20 september. Disponible en: https://bit.ly/3gLr6tG.

Consejo de la Unión Europea (2020c). Proposal for a Regulation of the European Parliament and of the Council establishing Horizon Europe, the Framework Programme for Research and Innovation, laying down its rules for participation and dissemination. Brussels, 18 December. Disponible en: https://bit.ly/3gKgrQ1.

Dewandre, N. (2002). European Strategies for Promoting Women in Science. Science, 5553, 278-279. Disponible en: https://doi.org/10.1126/science.1063487.

Díaz Martínez, C. y Samper Gras, T. (2014). Las mujeres y la ciencia: la apuesta por la investigación con perspectiva de género del Horizonte 2020.100cias@Uned, 7, 109-112. Disponible en: https://bit.ly/3vnBOuB.

Drew, E., Bencivenga, R., Ratzer, B., Poggio, B. y Saglamer, G. (2017). Gender in Horizon 2020: The Case of Gender Equality Plans. About Gender. International Journal of Gender Studies, 6 (12), 326-355.

European Institute for Gender Equality (2016a). Gender Equality in Academia and Research: GEAR Tool. Luxembourg. Disponible en: https://bit.ly/3u8REZC.

European Institute for Gender Equality (2016b). Promoting gender equality in academia and research institutions: Main findings. Luxembourg. Disponible en: https://bit.ly/2ScHf19.

European Institute for Gender Equality (2016c). Integrating gender equality into academia and research organisations: Analytical paper. Luxembourg. Disponible en: https://bit.ly/3sWipPH.

European Institute for Gender Equality (2016d). Gender in research. Luxembourg. Disponible en: https://bit.ly/2PvKL5R.

Genderaction (2018a). Gender in Horizon Europe. An Unfinshed Business. Briefing Paper, 1, June 2018. Disponible en: https://bit.ly/3exZ1n0.

Genderaction (2018b). Strengthening Gender in the ERA. Briefing Paper, 7, July 2018. Disponible en: https://bit.ly/3nuQHIA.

Genderaction (2018c). Gender-responsible Rules for Participation in Horizon Europe. Briefing Paper, 9, August 2018. Disponible en: https://bit.ly/3gHeFPL.

Gender-Net (2016). Manuals with guidelines on the integration of sex and gender analysis into research contents, recomendations for curricula development and indicators. Disponible en: https://bit.ly/3xx8BPx.

League of European Research Universities (LERU) (2018). Implicit bias in academia: A challenge to the meritocratic principle and to women's careers. And what to do about it. Advice Paper, 23. Disponible en: https://bit.ly/3vqObpL.

Leisyte, L. (2019). Gender in European Research Policy. En D. Simon et al. (ed.). Handbook on Science and Public Policy (pp. 284-315). London: Edward Elgar Publishing. Disponible en: https://doi.org/10.4337/9781784715946.00025.

Mergaert, L. y Lombardo, E. (2014). Resistance to implementing gender mainstreaming in EU research policy. European Integration online Papers (EIoP), 18 (5), 1-21. Disponible en: https://bit.ly/32OCkWa. 
Parlamento Europeo (2020). La igualdad entre hombres y mujeres. Fichas técnicas sobre la Unión Europea. Disponible en: https://www.europarl.europa.eu/ftu/ pdf/es/FTU_2.3.8.pdf.

Rees, T. (2001). Mainstreaming gender equality in science in the European Union: The «ETAN report». Gender and Education, 13 (3), 243-260. Disponible en: https://doi.org/10.1080/09540250120063544.

Sánchez, A., Samper Gras, T., Jabbaz, M. y Díaz Martínez, C. (2017). An open path for gender equality in research. When female scientists question the state of science and the institutions embrace the criticism. Mètode Science Studies Journal. 7, 97-103. Disponible en: https://doi.org/10.7203/metode.7.8187.

Standing Working Group on Gender in Research and Innovation (2018). Report on the implementation of Council Conclusions of 1 december 2015 on Advancing Gender Equality in the European Research Area. ERAC 1213/18. Brussels: European Research Area and Innovation Committee. Disponible en: https:// bit.ly/3eBG6YD.

Standing Working Group on Gender in Research and Innovation (2020a). Position paper on the future gender equality priority in the European Research Area 2020-2030. ERAC 1204/20. Brussels: European Research Area and Innovation Committe. Disponible en: https://bit.ly/3gH9qQ1.

Standing Working Group on Gender in Research and Innovation (2020b). Position paper on the current COVID-19 outbreak and gendered impacts on researchers and teachers. WK 5659/2020 INIT. Brussels: European Research Area Council. Disponible en: https://bit.ly/2RZJsg.

Vida, B. (2020). Policy framing and resistance: Gender mainstreaming in Horizon 2020. European Journal of Womens Studies, 1-16. Disponible en: https://doi. org/10.1177/1350506820935495. 